\title{
Hot Solvent Assisted Gravity Drainage in Naturally Fractured Heavy Oil Reservoirs: A New Model and Approach to Determine Optimal Solvent Injection Temperature
}

\author{
Joseph Sherratt, Amin Sharifi Haddad ${ }^{1}$, Roozbeh Rafati \\ School of Engineering, University of Aberdeen, SCO, UK
}

\section{Abstract}

2 Hot solvent assisted oil recovery is a low emission-intensity oil recovery method from heavy

3 oil resources. This method is particularly promising for fractured reservoirs where the

4 application of current thermal methods may involve challenges associated with heat loss and

5 early breakthrough. In this study a new model of heat and mass transfer for oil recovery from

6 a single matrix block of a naturally fractured reservoir using a hot miscible solvent is

7 developed. Due to the difference in magnitude between thermal and mass diffusivities, heat

8 diffuses beyond the solvent-oil interface and there is no significant convective heat transfer.

9 This results in a reduction of oil viscosity in the centre of the matrix block and a vertical convective flow pattern instead of parallel to the oil-solvent interface observed during cold solvent injection. Using this model optimisation graphs are developed to perform a fast qualitative assessment of the applicability of a hot solvent assisted gravity drainage process in naturally fractured reservoirs with various parameters without the need of complex simulations and experiments. An algorithm is presented to estimate the recovery time or target injection temperature of potential hot solvent assisted oil recovery processes using these optimisation graphs. This can reduce computational time and provide a quick evaluation of the hot solvent assisted gravity drainage process in naturally fractured heavy oil reservoirs.

Keywords: hot solvent; fractured reservoirs; heat conduction; solvent diffusion; convection; gravity drainage

\footnotetext{
${ }^{1}$ Corresponding author: Amin Sharifi Haddad, Email: amin.sharifi@abdn.ac.uk, Tel: +44 (0)1224 272977 Fax: +44 (4) 1224272497
} 


\section{Introduction}

High permeability fracture networks and high oil viscosities often exceeding one million $\mathrm{cP}$ reduce the efficiency of conventional and current thermal recovery methods from naturally fractured heavy oil and bitumen reservoirs often resulting in uneconomical recovery ${ }^{1}$. These types of reservoirs represent huge hydrocarbon resources that are only marginally exploited. The Canadian Grosmont formation alone is estimated to contain 406.5 billion bbl of heavy oil and bitumen in place ${ }^{2}$. Considerable efforts are being made to develop methods that could achieve economic oil recovery from these resources. The key to increasing recovery is a reduction of oil viscosity which is usually achieved by increasing the oil temperature or by oil dilution with a solvent.

A small increase in temperature has the potential to reduce the oil viscosity by several orders of magnitude ${ }^{3,4}$. Different methods such as in-situ combustion ${ }^{5}$, cyclic steam stimulation ${ }^{6}$, steam assisted gravity drainage (SAGD $)^{3,7,8}$, and their derivatives expanding solvent steamassisted gravity drainage $(\mathrm{ES}-\mathrm{SAGD})^{9-11}$, Steam-Over-Solvent $(\mathrm{SOS})^{12}$, Steam Alternating Solvent ${ }^{12}$ and the Solvent Aided Process $(\mathrm{SAP})^{13}$ have demonstrated promising solutions for unlocking and producing heavy oil from clastic reservoirs.

Adding a solvent achieves comparable viscosity reduction with a lower injection temperature than in pure thermal processes. Because the heat is usually generated via the combustion of fossil fuels, adding a solvent to the mixture has the added benefit of reducing the carbon emissions and cost associated with heat generation. However, it should be noted that injecting a solvent also has an associated cost (mainly due to purchasing the solvent) and the viscosity of the oil is only reduced when well mixed with the solvent. Many investigations have been performed that demonstrate the enhancement of oil and bitumen mobility by dilution with solvents. Zirrahi et al. in 2017 also showed that the presence of water in the reservoir can have a large impact on solvent solubility in bitumen thereby effecting the density and viscosity ${ }^{15,16}$. 
It has also been reported that the presence of asphaltenes can have an impact on solvent solubility in bitumen ${ }^{17}$.

Recently thermal solvent methods such as solvent-assisted SAGD (SA-SAGD) have been applied to real reservoirs and showed promising results ${ }^{18,19}$. Semi-analytical models have also been developed to model this process ${ }^{20}$. Leyva-Gomez and Babadagli in 2017 numerically modelled heavy oil recovery from oil sands with a high-temperature solvent which showed that the process is highly sensitive to temperature and pressure ${ }^{21}$. Their numerical studies also showed that a considerable amount of oil and solvent are left in the reservoir and other production processes should be considered to recover the residual hydrocarbons to increase efficiency and profit. Sabet et al. analysed the stability of the steam chamber-oil interface during the ES-SAGD process and suggested a fast screening method for the selection of an efficient solvent by evaluating the onset of convective mixing with bitumen ${ }^{22}$. Their model focused on the thermodynamic properties of solvents and the stability of the interface of the steam chamber. Similarly, the effects of phase behaviour on the SA-SAGD performance has been studied by Khaledi et al. concluding that a multicomponent hydrocarbon solvent may be used to significantly improve efficiency ${ }^{23}$. Marciales and Babadagli have also investigated the selection of optimal solvent discussing the need to find a compromise between low carbon number solvents that result in faster diffusion and high carbon number solvents that yield better mixing qualities but slower mixing ${ }^{24}$. However, the concepts of thermal-solvent recovery in fractured reservoirs has not been successfully modelled yet.

Electromagnetic heating of bitumen to reduce viscosity is another thermal method which has showed promising results through experimental and modelling studies ${ }^{25-28}$. However, based on previous studies this may only heat up a short distance from downhole electrical heaters. This 
The discussed processes may involve challenges when applied to fractured heavy oil reservoirs due to the high permeability contrast between matrix and fractures, which will result in a poor energy efficiency and early breakthrough. Therefore, studies have been conducted to explore solutions for heavy oil recovery from this type of reservoir over the last couple of years. Experimental studies have shown that hot solvent techniques have the potential to improve heavy oil recovery from fractured reservoirs but gave limited insight into the mechanisms that operate $^{24,29,30}$. Pathak et al. injected hot solvents into a cylinder containing preserved bitumen saturated cores (from the Grosmont formation) which were in contact with the solvent on all sides representing a matrix-fracture element of a fractured reservoir ${ }^{29}$. The solvent was allowed to soak into the core diluting the bitumen, then the mixture was collected from beneath the core. These experiments demonstrated that the hot solvent technique can deliver promising results even for highly viscous bitumen saturated carbonate reservoirs. Similar experiments have been conducted injecting liquid solvents into modified sandstone cores, to represent fractured reservoirs, concluding that lower injection rates result in higher recovery as the solvent has a longer time to diffuse into the bitumen before breakthrough occurs ${ }^{30}$. Gravity enhanced recovery using cold liquid solvents has also been explored. Kahrobaei et al. used a CT scanner to image solvent and oil saturations inside a matrix block during solvent assisted oil recovery and found that the type and properties of the oil and solvent influence the dominant mechanism of oil recovery ${ }^{31}$.

Recent investigations have shown that hydrocarbon solvent foams can increase the sweep efficiency while delivering solvent to the oil saturated regions of the reservoir ${ }^{32,33}$. It was found that foam bubbles in swept parts of the reservoir (the fractures) created resistance and diverted solvent towards the untouched regions (the matrix). The study also showed that $\mathrm{CO}_{2}$ foam and polymer enhanced foam can remarkably increase heavy oil recovery after solvent injection by diverting surfactant into the matrix. As foam is injected alongside the solvent the volume of 
required solvent is reduced. However, the foam stability at typical reservoir temperatures is poor where bubbles may collapse to form a continuous gas phase resulting in fast breakthrough. Increasing the foam quality and stability is essential to improving the efficiency of foam assisted oil recovery processes and is the focus of various experimental studies. These aim to find solutions for stable foams at reservoir conditions through additives such as polymers and nanomaterials $\mathrm{s}^{34,35}$.

There are a number of pilot schemes testing oil recovery methods in naturally fractured reservoirs through CSS, attempted steam drive and fire flooding among others. Edmunds et al. in 2009, reviewed previous pilots of the Grosmont formation concluding that reservoir complexity and areal heterogeneity impacted the efficiency of previous projects, especially steam and fire flooding ${ }^{36}$. They found that vertical permeability could exceed horizontal permeability and that gravity drainage methods could be an efficient alternative recovery method. This study also suggested that non-thermal solvent methods could result in promising recovery of bitumen from the matrix. The Saleski Pilot has confirmed that utilising gravity drainage methods, similar to SAGD in oil sands, can produce promising results in the Grosmont formation $^{37,38}$. In this pilot, it was also found that the high permeability of fractures introduces issues when producing in a cyclical scheme as the reservoir pressure declines during the soaking period and artificial lift may be required to produce the oil. The concept of cyclic-tocontinuous steam-assisted-gravity-drainage (C2C-SAGD) was developed to target bitumen in the fractures and vugs through cyclic steam injection and then continuous steam injection at the top of the reservoir targets bitumen in the matrix.

This has led to the emergence of thermal-solvent methods where a hot solvent is injected as or alongside the heating agent instead of alternating steam and solvent injections. Liquid phase heating agents should result in later breakthrough than gaseous phases such as steam resulting in a more efficient sweeping and heating of the fractured reservoir. The dual actions of dilution 
and heating of the oil work together to reduce the oil viscosity. Sharifi Haddad and Gates showed that in post-CHOPS reservoir where the formation includes high permeability wormholes the use of hot water and $\mathrm{CO}_{2}$ can improve oil recovery through thermal and dilution processes. They showed that thermally assisted $\mathrm{CO}_{2}$ injection processes provide superior performance compared to water flooding or $\mathrm{CO}_{2}$ injection while eliminating the gas breakthrough issues ${ }^{39}$.

Recovery rates from fractured reservoirs are controlled by the rate of mass transfer between the matrix and the fractures. The use of a miscible injected phase may be a favourable process when capillary forces oppose spontaneous imbibition of water or gas into the matrix. Low permeability matrix blocks often result in diffusion dominated mass transfer within the matrix blocks and exponentially declining recovery rates $^{40-42}$. Gravity forces due to the difference in density between the injected solvent and oil should also help increase convective mass transfer, thereby increasing recovery rates.

Modelling thermal and non-thermal oil recovery from fractured reservoir has been mainly performed using the dual porosity approach where the majority of the oil is stored in low permeability matrix blocks and the convective mass transfer occurs in fractures ${ }^{43}$. Heat diffusion is usually quicker than the mass (solvent) diffusion by at least an order of magnitude. This makes the modelling of the process complex as a dual boundary layer problem is created. In the first boundary layer both dilution and heating of the oil act to lower the viscosity of the mixture. Beyond this boundary, only the heating process acts to lower the viscosity of the pure oil. Analytical models corresponding to this dual action process have been produced to calculate recovery in sandstone reservoirs ${ }^{10,44}$.

Models for oil recovery from fractured reservoirs considering pure steam injection and cold solvent injection have been developed in previous studies ${ }^{31,45-47}$. Kahrobaei et al. attempted to simulate the oil recovery from a core sample under solvent assisted gravity drainage process 
using commercially available simulators. However, in order to match their experimental data unrealistically low mass diffusion coefficients had to be used $^{31}$. Okazawa proposed that diffusion of solvent into heavy oil can be a concentration dependent process ${ }^{45}$. Thereafter, Sharifi Haddad et al. showed that the use of a concentration dependant mass diffusion coefficient for solvent assisted oil recovery from fractured reservoirs can improve the accuracy of the models ${ }^{46}$.

Since the concept of simultaneous heat and mass diffusion processes has not been considered in fractured reservoirs before, in this study we extend the previous model proposed by Sharifi Haddad et al. by incorporating the concept of heat transfer. A model is presented to understand the mechanisms of heavy oil recovery from a single matrix block of a fractured reservoir using a hot solvent assisted oil recovery process. A concentration dependant mass diffusion coefficient is essential to match such models with experimental data and should be used in this study. The model is developed through the coupling of heat, mass and momentum equations. It is showed that simulation of such processes are computationally expensive. Optimisation graphs were developed to analyse the performance of hot solvent injection in naturally fractured reservoirs under a range of different conditions without the use of complex reservoir simulators. An optimisation algorithm is also presented which can be used with the optimisation graphs for the design of the hot solvent assisted oil recovery processes from heavy oil fractured reservoirs.

Due to the cost of solvents the pure hot solvent process is unlikely to be economically viable as a recovery process. It is most likely that in real field applications another fluid will be injected to reduce the volume of solvent required; such as the steam-over-solvent and steam alternating solvent process where steam and solvent are alternatively injected ${ }^{12,13}$. The optimisation algorithm presented in this study could be used to understand the effectiveness of different solvents and required injection temperatures in these scenarios. 


\section{Mathematical Model}

In this study, we assume a 3-D matrix block is surrounded by three sets of fracture planes in a fractured reservoir. Initially, the low permeability matrix block is saturated with heavy oil. Due to the high permeability of the fractures it is assumed that they will quickly become saturated with the injected miscible solvent. At the interface of fracture and matrix block, solvent and heat diffuse into the matrix block lowering the viscosity. This produces a mixed region where gravity driven convective mass and heat transfer can occur. It is expected that once oil/oilsolvent mixture drains into the fractures it flows away quick enough that the solvent concentration in the fracture remains constant. It is further assumed that the solvent and bitumen are fully miscible, form a binary mixture through ideal mixing and that there is no connate water present.

The mass transfer process is a combination of convection and diffusion and expressed in the general form as Equation (1) assuming that the fluids and rock matrix are incompressible.

$\phi \frac{\partial C}{\partial t}=\boldsymbol{\nabla} \cdot\left(D_{e} \boldsymbol{\nabla} C\right)-\boldsymbol{\nabla} \cdot(\boldsymbol{U} C)$

Where $C$ is the volumetric solvent concentration, $t$ is time, $\phi$ is porosity and $\boldsymbol{U}$ is the flow velocity vector. In a porous media the effective diffusion coefficient, $D_{e}$ given by Equation (2), is used as it takes into account the porosity $\phi$, pore constrictivity $\delta$, the pore tortuosity $\tau$ and $D_{m}$ is the solvent-oil molecular diffusion coefficient ${ }^{48}$.

$$
D_{e}=\frac{D_{m} \phi \delta}{\tau}
$$

Previous modelling attempts have shown that the mass diffusion coefficient should be represented by a concentration dependant relationship ${ }^{45,46} . D_{m}$ in Equation (2) can be replaced by the concentration dependant expression, $D(C)$, as shown in Equation (3).

$$
D(C)=D_{m}\left(\frac{C}{C_{f}}\right)^{n}=D_{m} C_{D}^{n}
$$


192

193

194

195

$$
\boldsymbol{U}=\frac{-\boldsymbol{k}}{\mu_{\text {mix }}}\left(\boldsymbol{\nabla} P+\rho_{\text {mix }} \boldsymbol{g}\right)
$$

$$
\boldsymbol{k}=\left(\begin{array}{l}
k_{x} \\
k_{y} \\
k_{z}
\end{array}\right)
$$

$204 \boldsymbol{g}=\left(\begin{array}{l}0 \\ 0 \\ g\end{array}\right)$

Where $P$ is the pore fluid pressure, $\mu_{m i x}$ is the fluid mixture viscosity, $\rho_{m i x}$ is the fluid mixture density and $g$ is acceleration due to gravity. The matrix domain is assumed to be homogeneous and isotropic which reduces the permeability vector $\mathbf{k}$ to the scalar value $k$.

208 Previous studies have assumed that oil and solvent mix ideally ${ }^{31,46}$. This has been confirmed experimentally using aromatic solvents ${ }^{49}$. Other studies have shown that ideal mixing is not always observed. Luo et al. concluded that volume changes are significant and as a result the

211 rate of mass diffusion is reduced ${ }^{50}$. However, in their study the maximum volume change 
reported was approximately $2 \%$. This may require further investigations to understand whether the volume change during mixing affects the diffusion coefficient, or if it is mainly the concentration that influences the diffusion coefficient. To avoid introducing another unknown variable in this study the assumption of ideal mixing is made and the mixture density is expressed using the volume fraction relationship as in Equation (8) where $V_{S}$ and $V_{o}$ are volume fractions of solvent and oil respectively. This can be expressed using the volumetric solvent concentration, $C$, in Equation (9).

$\rho_{\text {mix }}=\rho_{s} V_{s}+\rho_{o} V_{o}$

$\rho_{\text {mix }}=\rho_{s} C+\rho_{o}(1-C)$

Where $\rho_{o}$ and $\rho_{s}$ are the oil and solvent densities. This can be expressed in a dimensionless form by dividing by $\rho_{o}$ as in Equation (11) and $\rho_{D}$ is given by Equation (12). As this study assumes the injection of pure solvent then $C$ can also be replaced with $C_{D}$.

$\rho_{\text {mix }}=\rho_{o} \rho_{\text {mix }, D}$

$\rho_{\text {mix }, D}=\rho_{D}\left(C_{D}+\left(1-C_{D}\right) \frac{1}{\rho_{D}}\right)$

$\rho_{D}=\rho_{s} / \rho_{o}$

The mixture viscosity can be expressed by combining the Lederer and Butler relationships to account for both solvent concentration and temperature as shown in Equation $(13)^{20}$, and the exponent $X_{S}$ is given by Equation (14).

$\mu_{m i x}=\mu_{o, h o t}\left(\frac{\mu_{s}}{\mu_{o, h o t}}\right)^{X_{S}}\left(\frac{T-T_{R}}{T_{s}-T_{R}}\right)^{m}$

$X_{S}=\frac{C_{D}}{\beta\left(1-C_{D}\right)+C_{D}}$ 
232

233

234

235

236

237

238

239 Where,

$240 \quad \mu_{D}=\frac{\mu_{o, R}}{\mu_{s}}$

$241 \quad \mu_{T}=\frac{\mu_{o, R}}{\mu_{o, h o t}}$

$242 \quad T_{D}=\frac{T-T_{R}}{T_{f}-T_{R}}$

243 The dimensionless thermal viscosity reduction term $\mu_{T}$ is defined as the ratio between oil

244 viscosity at initial and injected solvent temperature.

245 The pressure can be expressed in a dimensionless form, $P_{D}$ as Equation (19) where $L$ is the 246 characteristic length of the matrix block.

$247 \quad P_{D}=\frac{P}{\rho_{o} g L}$

248 Therefore, the mass transport equation can be given in a dimensionless form as Equation (20)

249 in dimensionless coordinates $x_{D}, y_{D}$ and $z_{D}$, and $\boldsymbol{e}_{k}$ is the unit normal vector in the z-direction.

$250 \quad \frac{\partial C_{D}}{\partial t_{D}}=\boldsymbol{\nabla} \cdot\left(\mathrm{C}_{D}^{n} \nabla \mathrm{C}_{D}\right)+P e \boldsymbol{\nabla} \cdot\left(\frac{C_{D}}{\mu_{m i x, D}}\left(\boldsymbol{\nabla} P_{D}+\rho_{m i x, D} \boldsymbol{e}_{\boldsymbol{k}}\right)\right)$ 
251 Where,

$252 P e=\frac{g k L}{D_{s-o} v_{o, R} \phi}$

$253 \quad t_{D}=\frac{D_{s-o} t}{L^{2}}$

$254 \quad D_{s-o}=\frac{D_{e}}{\phi}=\frac{D_{m} \delta}{\tau}$

$255 \quad x_{D}=\frac{x}{L}$

$256 \quad y_{D}=\frac{y}{L}$

$257 \quad Z_{D}=\frac{z}{L}$

258 Where the Péclet number, $P e$, represents the ratio of gravity assisted convective transfer to 259 diffusive mass transfer, and $v_{o}$ is the kinematic oil viscosity. The initial and boundary 260 conditions are as below and $C_{m, D}$ represents the solvent concentration at any point in the matrix.

$261 C_{f}=1$ when $t \geq 0$

$262 C_{D}=\left\{\begin{array}{r}0, t=0 \\ 0 \leq C_{m, D} \leq 1, t>0\end{array}\right.$

263 The pressure field can by calculated by applying the non-divergent flow field condition,

264 Equation (29).

$265 \nabla \cdot \boldsymbol{U}=0$

266 This can be expressed in a dimensionless form as Equation (30).

$267 \boldsymbol{\nabla} \cdot\left(\frac{1}{\mu_{m i x, D}}\left(\boldsymbol{\nabla} P_{D}+\rho_{m i x, D} \boldsymbol{e}_{\boldsymbol{k}}\right)\right)=0$ 
$\frac{\partial}{\partial t}\left(C_{B, D}^{*} T\right)=\nabla \cdot\left(\lambda_{e} \nabla \mathrm{T}\right)-\nabla \cdot\left(\boldsymbol{U} C_{m i x, D}^{*} T\right)$ and $h_{D}$ is the dimensionless depth in the fracture.

$P=P_{r e f}+\rho_{s} g h$

$P_{D}=\rho_{D} h_{D}$ principle of conservation of heat as in Equation (33). solvent mixture is given by Equation (35).

$\frac{C_{B}^{*}}{\phi}=\frac{(1-\phi)}{\phi} C_{\text {rock }}^{*}+C C_{s}^{*}+(1-C) C_{o}^{*}$

$C_{m i x}^{*}=C C_{s}^{*}+(1-C) C_{o}^{*}$

The initial pressure in the fracture and matrix is assumed to be hydrostatic. It is further assumed that the solvent concentration in the fracture is constant and therefore the pressure in the fracture is also constant. Therefore, the fracture pressure at any depth, h, beneath the top of the matrix block is given by Equation (31). Assuming that the pressure at the top of the matrix, $P_{r e f}=0$, this can be expressed in a dimensionless form as Equation (32) by dividing by $\rho_{o} g L$,

The heat transfer equation can be formed by combining Fick's law of heat diffusion and the

Where $C_{B, D}^{*}$ and $C_{m i x, D}^{*}$ are the bulk and liquid mixture volumetric heat capacities respectively, $T$ is the temperature, $\lambda_{e}$ is the effective thermal conductivity. It is assumed that any point inside the matrix is in a state of isothermal equilibrium between the solid and liquid. The volumetric heat capacity of a volume of the matrix domain can be determined from the volume weighted approach in Equation $(34)^{51,52}$. In addition to this the volumetric heat capacity for the oil-

Where $C_{\text {rock }}^{*} C_{o}^{*}$ and $C_{s}^{*}$ are the volumetric heat capacities of rock, oil and solvent respectively which is the product of the specific heat capacity and density of each pure substance, Equation (36). These can be expressed in a dimensionless form by dividing by $C_{o}^{*}$. 
$291 \quad C_{B, D}^{*}=\frac{C_{B}^{*}}{\phi C_{o}^{*}}$

$292 C_{m i x, D}^{*}=\frac{C_{m i x}^{*}}{C_{o}^{*}}$

293 The effective thermal conductivity can be calculated assuming a serial connection, Equation

294 (39) and expressed in a dimensionless form as Equation (40) by dividing by $\lambda_{o}$.

295

$\lambda_{e}=(1-\phi) \lambda_{\text {rock }}+\phi C \lambda_{s}+\phi(1-C) \lambda_{o}$

296

$$
\lambda_{e, D}=\frac{\lambda_{e}}{\lambda_{o}}=(1-\phi) \frac{\lambda_{\text {rock }}}{\lambda_{o}}+\phi C \frac{\lambda_{s}}{\lambda_{o}}+\phi(1-C)
$$

297 Therefore, the dimensionless heat transfer equation is given by Equation (41).

$298 \frac{\partial}{\partial t_{D}}\left(C_{B, D}^{*} T_{D}\right)=L e \nabla \cdot\left(\lambda_{e, D} \nabla \mathrm{T}_{D}\right)+P e \nabla \cdot\left(\frac{C_{m i x, D}^{*} T_{D}}{\mu_{m i x, D}}\left(\nabla P_{D}+\rho_{m i x, D} \boldsymbol{e}_{k}\right)\right)$

299 Where,

300

$L e=\frac{\lambda_{o}}{\phi D_{S-o} C_{o}^{*}}$

301

302

303

304

305

306

307

308

309

$L e$ is the Lewis number which represents the dimensionless ratio of thermal diffusivity to mass diffusivity.

The system of coupled partial differential equations (Equations 20, 30, and 41) are solved numerically using a Finite Volume Method (the discretised equations and algorithm used are detailed in the appendix). The pressure field, $P_{D}$, within the matrix domain can be solved at each time step with known solvent concentration and temperature fields, $C_{D}$ and $T_{D}$. Then a velocity field at each time step can be calculated, which can be used to solve for solvent concentration $C_{D}$, and temperature $T_{D}$, implicitly at the next time step. Each system of equations has the form $\boldsymbol{A x}=\boldsymbol{b}$ where $\mathbf{A}$ is a sparse $\mathrm{n}$ by $\mathrm{n}$ heptadiagonal matrix and $\mathrm{n}$ is the number of 
cells in the model. A program developed using a $\mathrm{C}++$ programing platform was used to solve these equations using the conjugate gradient method to solve the system of equations ${ }^{53}$.

\section{Results and discussion}

The model developed can be used to study the mass transfer mechanisms during isothermal solvent injection, where oil dilution alone reduces viscosity, or during hot solvent injection where both oil dilution and heat reduce viscosity. A sensitivity study of the variables shows how the system responds to different rock and fluid properties. The response of the system can then be studied using a hot injected solvent and another sensitivity study shows the response of the system to different thermal properties.

\subsection{Isothermal solvent injection}

The injected solvent will always be more mobile than the native oil. However, due to the matrix permeability the solvent may have high or low mobility. In a system with high solvent mobility but low oil mobility due to high viscosity, a drainage profile as shown in Figure 1 is observed where $P e=10, \mu_{D}=1000, \rho_{D}=0.6, n=2$. The drainage profiles show that initially the solvent diffuses into the matrix block. Once there is a solvent-oil mixture with intermediate viscosity and density, gravity initiates the convective flow in the matrix block toward the fractures. This figure also shows Raleigh-Taylor instabilities underneath the oil saturated region which occur when a more dense fluid is situated above a less dense fluid ${ }^{54,55}$. These will help to increase the rate of recovery. A convective dominated system will result in high recovery rates which is more likely to be economically feasible. Therefore, it is important to understand the behaviour of the system under different conditions. 


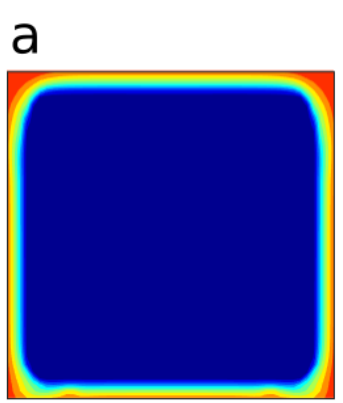

b

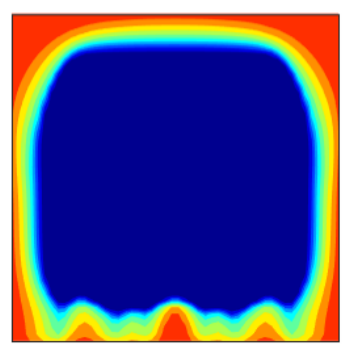

C

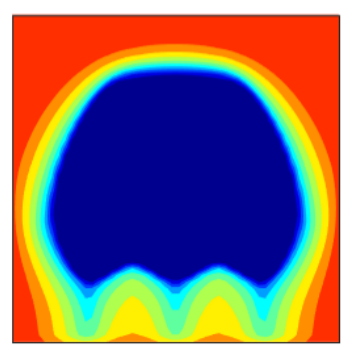

d

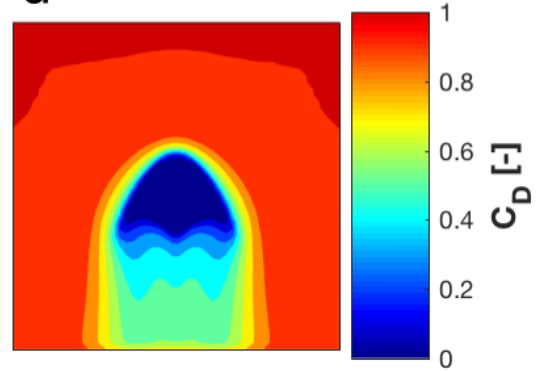

Figure 1 - Solvent concentration profile through the centre of the model (vertical plane) when oil mobility is low but solvent mobility is high at a) $\left.t_{D}=0.002, b\right) t_{D}=0.005, c$ ) $t_{D}=0.01$ and d) $t_{D}=0.02$ for $P e=10, \mu_{D}=1000, \rho_{D}=0.6 \& n=2$.

As shown in Figure 1, the Raleigh-Taylor instabilities start at small wavelength perturbations (a-b) which coalesce and grow into larger wavelength features (c). At this point, the finger beneath the centre of the oil saturated region remains fixed but as the oil drains the fingers on the edge of the oil saturated region move inwards. This has the effect of reducing the wavelength of these perturbations (c-d). These instabilities are only seen when the solvent is highly mobile and the oil is not which is representative of high permeability and high oil viscosity with low solvent viscosity.

To determine the number of cells and time step size to be used in the rest of this study a sensitivity analysis was performed to ensure numerical errors are minimised. The isothermal model was tested because the drainage pattern is the most complex and should therefore require a finer grid and time step size than the thermal model. Figure 2a shows that decreasing the time step from $1 \times 10^{-6}$ to $1 \times 10^{-7}$ has negligible effect on the recovery curve. Figure $2 \mathrm{~b}$ shows that increasing the number of cells from $50 \times 50 \times 50$ to $75 \times 75 \times 75$ has a very small effect on the recovery curve. The program was run on a single $2.20 \mathrm{GHz}$ core of the Maxwell HPC and the $75 \times 75 \times 75$ cell model took 136 hours to run with a time step size of $1 \times 10^{-6}$. To increase computational efficiency a model with $50 \times 50 \times 50$ cells and time step size of $1 \times 10^{-6}$ may be used to reduce the runtime to under 24 hours without significantly impacting the accuracy of the model. 

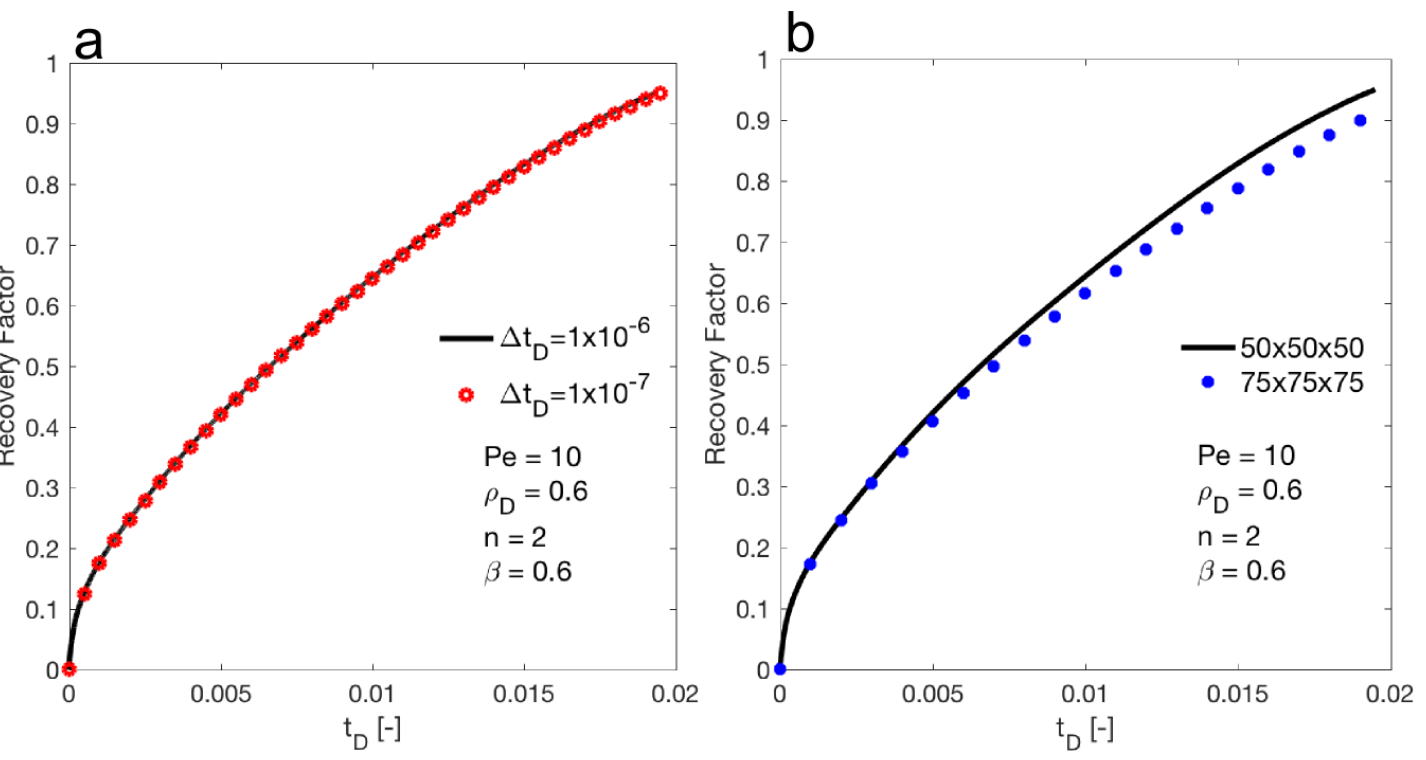

Figure 2 - Sensitivity analysis showing the response of the isothermal model with a) different 355 time step sizes using a 50×50×50 cell model. b) different numbers of cells in the model using 356 a time step size of $1 \times 10^{-6}$.

High Péclet numbers represent high permeability, high oil density and low oil viscosity. As the Péclet number increases the system becomes more convective and therefore recovery time decreases. During initial stages, recovery is diffusion dominated, and the Péclet number only has an effect on oil recovery at later times. A low Péclet number represents a system where permeability is very low and the oil is highly immobile, and therefore mass transfer is diffusion dominated. A high viscosity ratio $\mu_{D}$ represents a low solvent viscosity or a high oil viscosity. As the solvent viscosity decreases the oil-solvent mixture becomes more mobile and therefore mass transfer becomes more convective which reduces oil recovery time. The convective flow is entirely driven by gravity and therefore large differences in the density between the oil and the solvent increases the convective behaviour of the system and increases the oil recovery rate. If the solvent mobility is low then the density ratio will have little to no effect on the behaviour of the system and if there is no difference in density the mass transfer is entirely diffusive as there is no potential to drive convective flow.

The concentration dependant diffusion exponent, $n$, is dependent on the specific solvent-oil system and determined experimentally. Larger values of $n$ decrease the thickness of the mixed 
zone between the oil and solvent saturated regions where convective flow occurs. By narrowing this region where oil viscosity is reduced, at any time less volume of fluid is free to flow, thereby reducing oil recovery rates.

\subsubsection{Validation of isothermal model}

The isothermal model can be validated against available data for laboratory experiments performed using high permeability core samples saturated with oil and submerged in a miscible solvent. Data from two sets of experiments have been used with specific oil and solvent properties shown in Table $1^{31}$. Oil saturation inside the core plug was measured using a CT scanner throughout the experiment. The original study attempted to replicate the experiment using MoReS (Shell in-house reservoir simulator) but failed to replicate the experiment using realistic mass diffusion coefficients calculated using the Wilke-Chang equation ${ }^{56}$, Equation (43). Where the association factor $\Phi$ is assumed to be 1 for unassociated materials, $M_{S}$ is the molecular mass of the solvent, $T$ is the temperature in Kelvin, $\mu_{o}$ is the oil viscosity and $V_{S}$ is the molar volume of the solvent.

$D_{m}=\frac{7.4 \times 10^{-12}\left(\Phi M_{S}\right)^{0.5} T}{\mu_{o} V_{s}^{0.6}}$

This gave the model parameters as in Table 1 . The term $\delta / \tau$ is assumed to be 1 as the pore geometry is unknown. A one-dimensional model developed by Sharifi Haddad et al. was able to capture the behavior of one of the experiments with the core sample that showed a one dimensional drainage pattern ${ }^{46}$. A mass diffusion exponent of 2 was confirmed to give a good match between their model and experimental data. The only variation is the use of the concentration dependant mass diffusion coefficient.

In this study, the cylindrical core plugs used in the experiments ${ }^{31}$ were represented in the model by a cuboidal block of dimensionless height and width of 1 and 0.63 respectively, with a characteristic length of $L=6 \times 10^{-2} \mathrm{~m}$. This conserved the surface area to volume ratio of the 
core plug. For both experiments the block was represented by a $40 \times 40 \times 50$ cell model. The

397

398

399 400

401

402

403

404

405

406

407

number of cells was reduced as the geometry of the domain was changed. Since the size of the cells was not increased there should not be any impact on the accuracy of this model.

Table 1 -Model parameters for experiment $1 \& 2$ using the mass diffusion coefficient calculated using Equation (42) ${ }^{31}$.

\begin{tabular}{lllcccc}
\hline Exp. & \multirow{2}{*}{ Oil } & Solvent & $\begin{array}{c}\rho_{D} \\
{[-]}\end{array}$ & $\begin{array}{c}\mu_{D} \\
{[-]}\end{array}$ & $\begin{array}{c}D_{m} \\
\left(\mathbf{m}^{2} / \mathbf{s}\right)\end{array}$ & $\begin{array}{c}P e \\
{[-]}\end{array}$ \\
\hline 1 & \multirow{2}{*}{ Pentane } & Declain & 1.43 & 0.07 & $5.9 \times 10^{-9}$ & 1673 \\
\hline 2 & \multirow{2}{*}{ Hexadecane } & Declain & 1.17 & 1.05 & $3.3 \times 10^{-10}$ & 2700
\end{tabular}

The results shown in Figure 3 show that this model has a close match with the experimental results. The main source of error is likely to result from using a cuboidal block in the model whereas the core plugs are cylindrical. In addition, the boundary condition of the model assumed solvent concentration in the fractures is constant which was not exactly the same condition in the experiments as a fixed volume of solvent was inside the container where the core was submerged. However, the volume of solvent was very large compared to the oil volume inside the core, which makes our assumption reasonable.
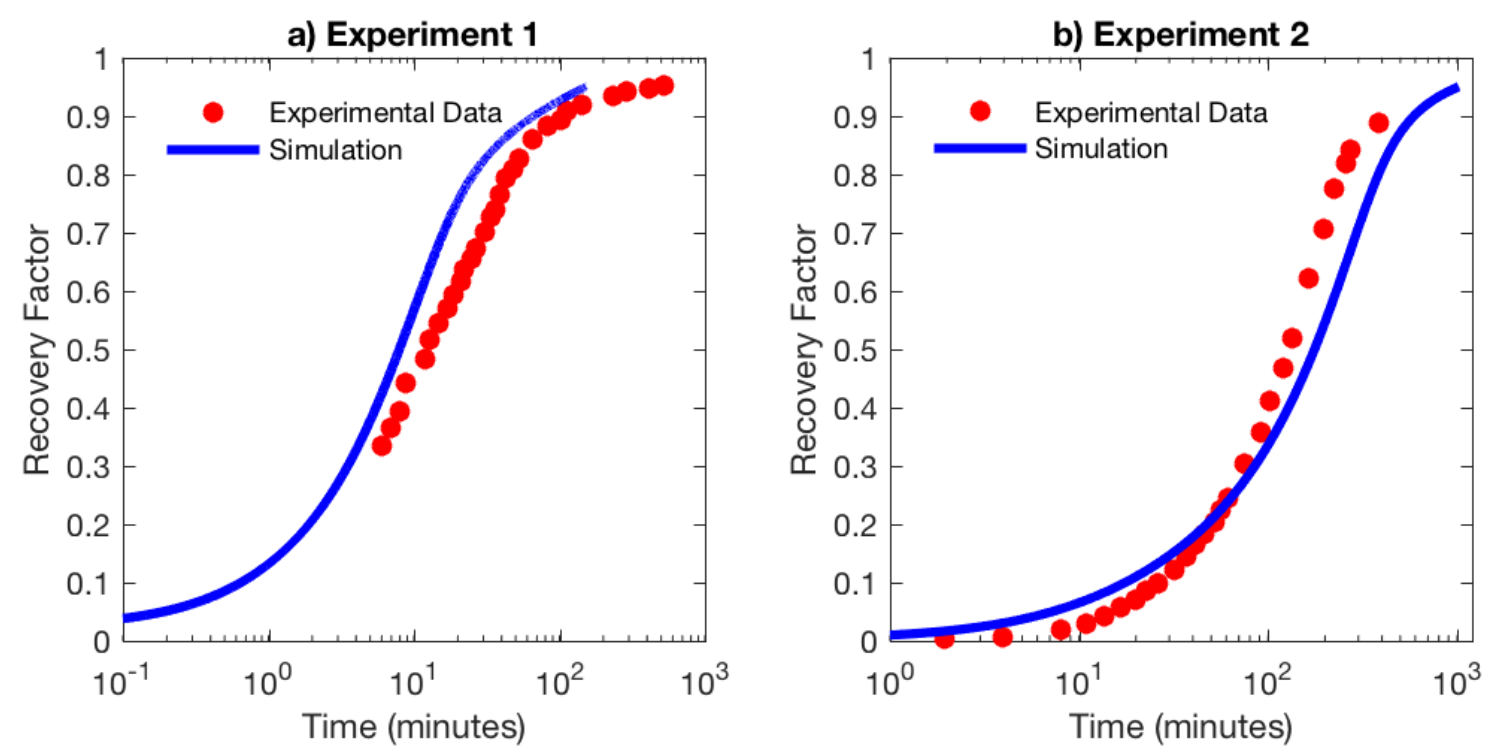

408

Figure 3 - Comparison of experimental ${ }^{31}$, and simulation recovery profiles (this study) for 410 experiment 1 and 2. 
411 Solvent concentration profiles of a slice through the centre of the core plug are shown at various

412 times during the experiment measured from CT scans and compared with a slice through the

413 centre of the model in Figure 4 and Figure 5 for experiment 1 and 2 respectively. The

414 concentration profiles show a similar behaviour between the model and the experiment in both

415 cases. As shown in Figure 3 the simulated recovery is a little behind the recorded recovery for

416 experiment 2 at later times, confirmed in Figure 5, and is most likely a result of the cubic core

417 plug representation.

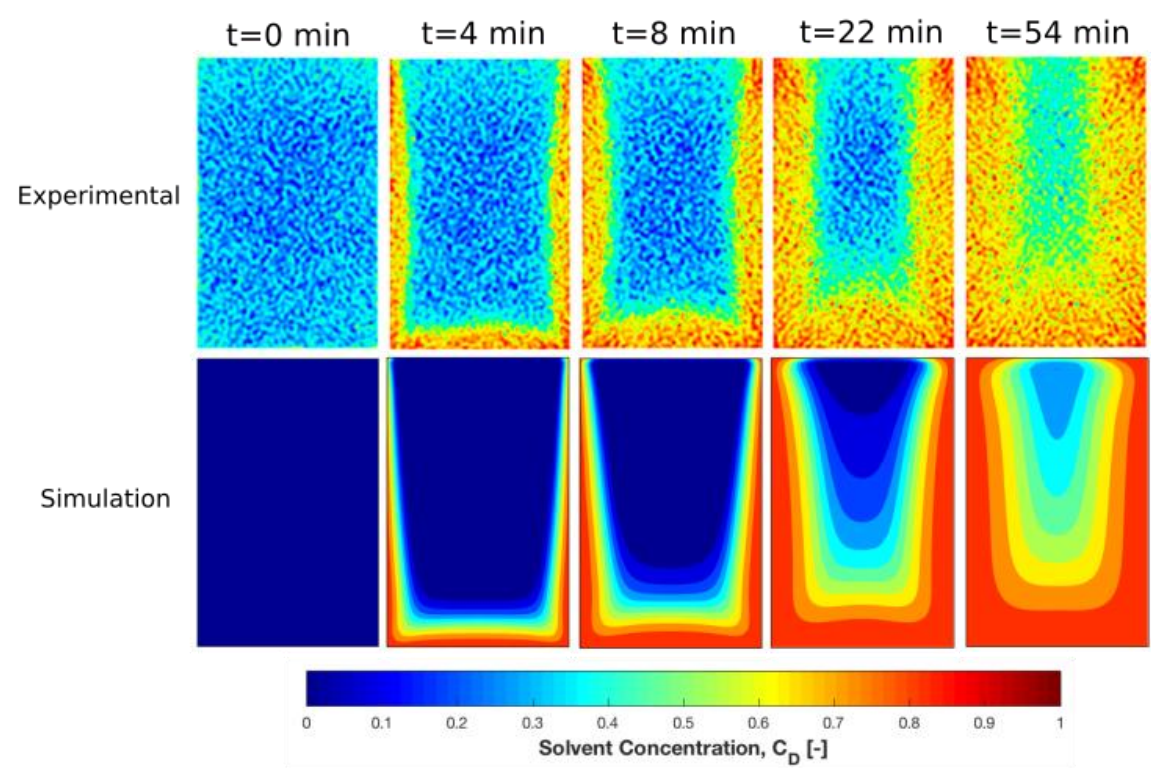

419 Figure 4-Comparison of solvent concentration profile through the centre of the core plug 420 from the experiment ${ }^{31}$, and the model (this study) for experiment 1. 


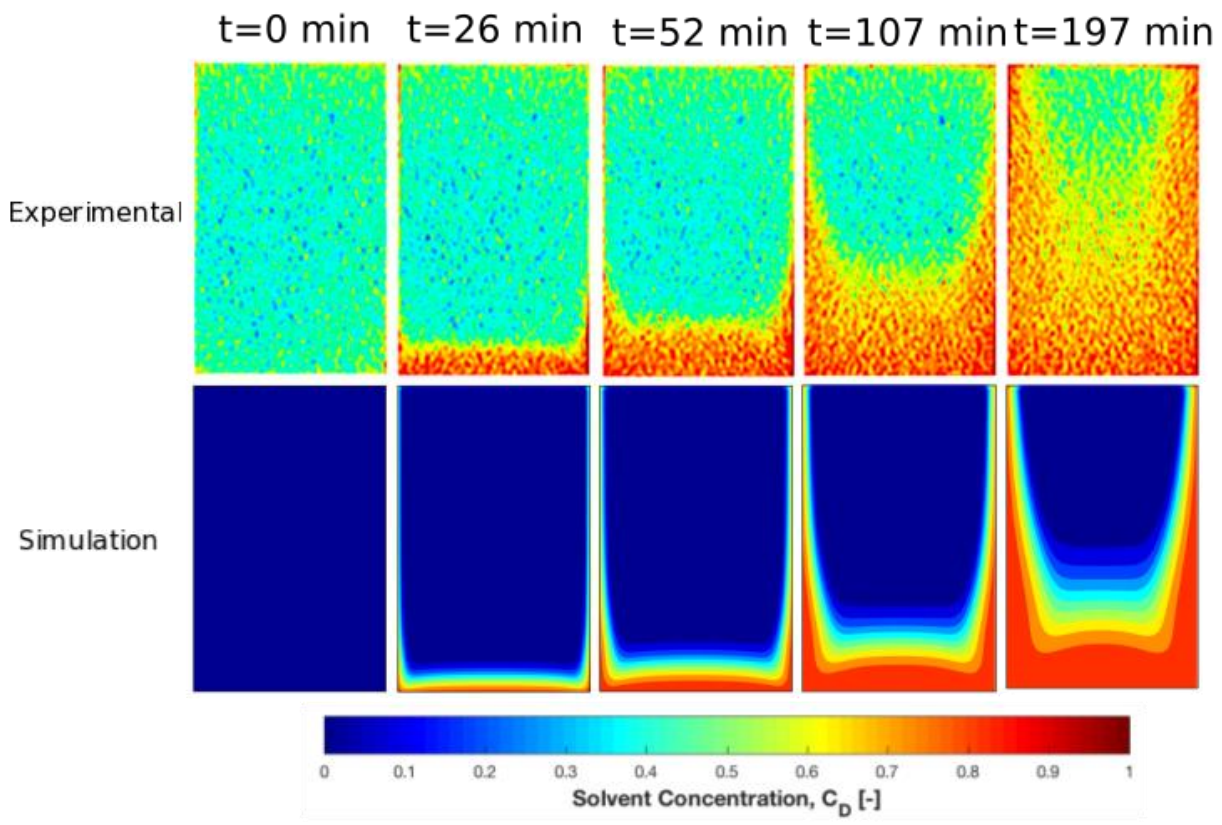

422 Figure 5 -Comparison of solvent concentration profile through the centre of the core plug 423 from the experiment ${ }^{31}$, and the model (this study) for experiment 2.

\subsection{Hot solvent injection}

In this study, simultaneous heat and mass transfer during the oil recovery processes from fractured reservoirs was modelled. Therefore, in the rest of this study we focus on the mechanisms of hot solvent assisted oil recovery and optimisation of such processes in fractured reservoirs using the solutions of our model. The reduction of oil viscosity with temperature is captured in the model by increasing the dimensionless thermal viscosity reduction term, $\mu_{T}$. This value can be found for any system when the oil properties and injection temperature are known. Modelling of a hot solvent assisted oil recovery process requires further information regarding the thermal properties of the system. The dimensionless values used are summarised in Table 2 which are calculated from the values in Table 3 and porosity, $\phi=0.2$. The thermal properties of the system are unlikely to change greatly with different solid and fluid samples.

Table 2 -Dimensionless thermal properties of fluid and rock

\begin{tabular}{cccc}
\hline $\begin{array}{c}\text { Dimensionless } \\
\text { Property }\end{array}$ & Rock & Oil & Solvent \\
\hline$C^{*}{ }_{D}[-]$ & 1.15 & 1.0 & $\rho_{D}$
\end{tabular}




\begin{tabular}{llll}
\hline$\lambda_{D}[-]$ & 16.67 & 1.0 & 1.0
\end{tabular}

436

437 Table 3 - Thermal properties of rock and fluids

$\begin{array}{ll}\text { Rock specific heat capacity } & 850 \mathrm{JK}^{-1} \mathrm{~kg}^{-1} \\ \text { Oil specific heat capacity } & 2000 \mathrm{JK}^{-1} \mathrm{~kg}^{-1} \\ \text { Solvent specific heat capacity } & 2000 \mathrm{JK}^{-1} \mathrm{~kg}^{-1} \\ \lambda_{\text {rock }} & 2.5 \mathrm{Wm}^{-1} \mathrm{~K}^{-1} \\ \lambda_{s} & 0.15 \mathrm{Wm}^{-1} \mathrm{~K}^{-1} \\ \lambda_{o} & 0.15 \mathrm{Wm}^{-1} \mathrm{~K}^{-1} \\ \rho_{o} & 1000 \mathrm{kgm}^{-3} \\ \rho_{\text {rock }} & 2700 \mathrm{kgm}^{-3}\end{array}$

438 Figure 6 shows that using hot solvent with a high value of $\mu_{T}$ can greatly reduce recovery time.

439 With very high injection temperatures, the rate of recovery remains roughly constant compared 440 to the cold solvent case where the rate of recovery declines over time ( $\mu_{T}=1$ shows the cold 441 solvent case).

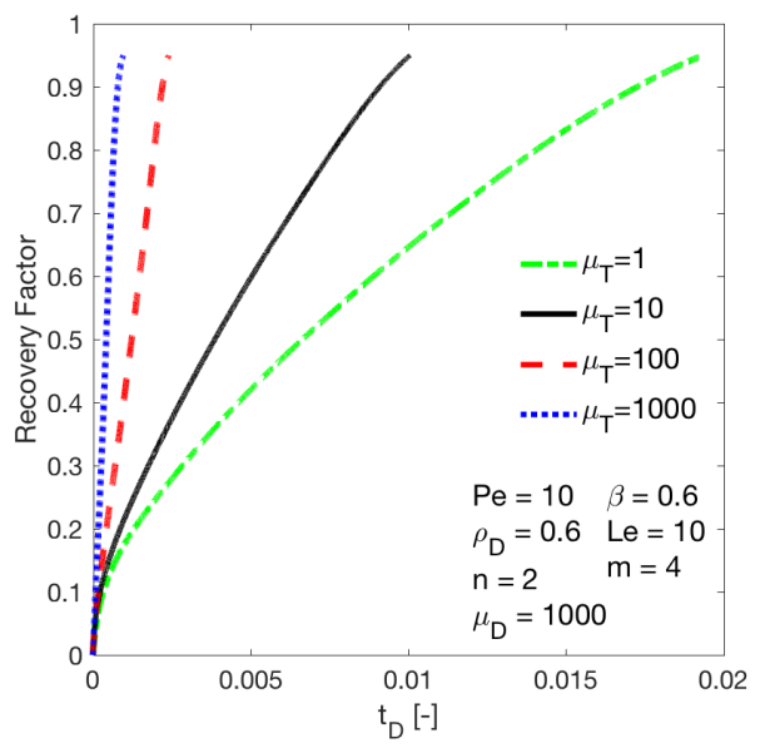

443 Figure 6 - Recovery curves for different values of $\mu_{T}$ representing different temperatures of 444 injected solvent. 
Figure 7 shows the drainage pattern when a hot solvent is used and demosntrates that hot solvent assisted oil recovery creates a different drainage profile than cold solvent assisted oil recovery as shown is Figure 1, having all other parameters the same. The temperature of the oil is increased much beyond the solvent-oil interface reducing the viscosity. This means that the oil can move together as a single body towards the bottom fracture. This is shown in Figure 8 which clearly demonstrates that for cold oil flow velocity in oil saturated regions is negligable and all flow occurs parallel to the solvent-oil interface in the mixed zone. In contrast, there is a high flow velocity far away from the solvent-oil interface in the centre of the matrix block using a hot solvent. Also it can be seen that for the hot solvent case, the flow is vertical instead of parallel to the oil-solvent interface. This shows that heat transfer is a diffusion dominated process and it can enhance the mixing at the interface of oil and solvent, which eventually helps to have convective flow in the matrix block.
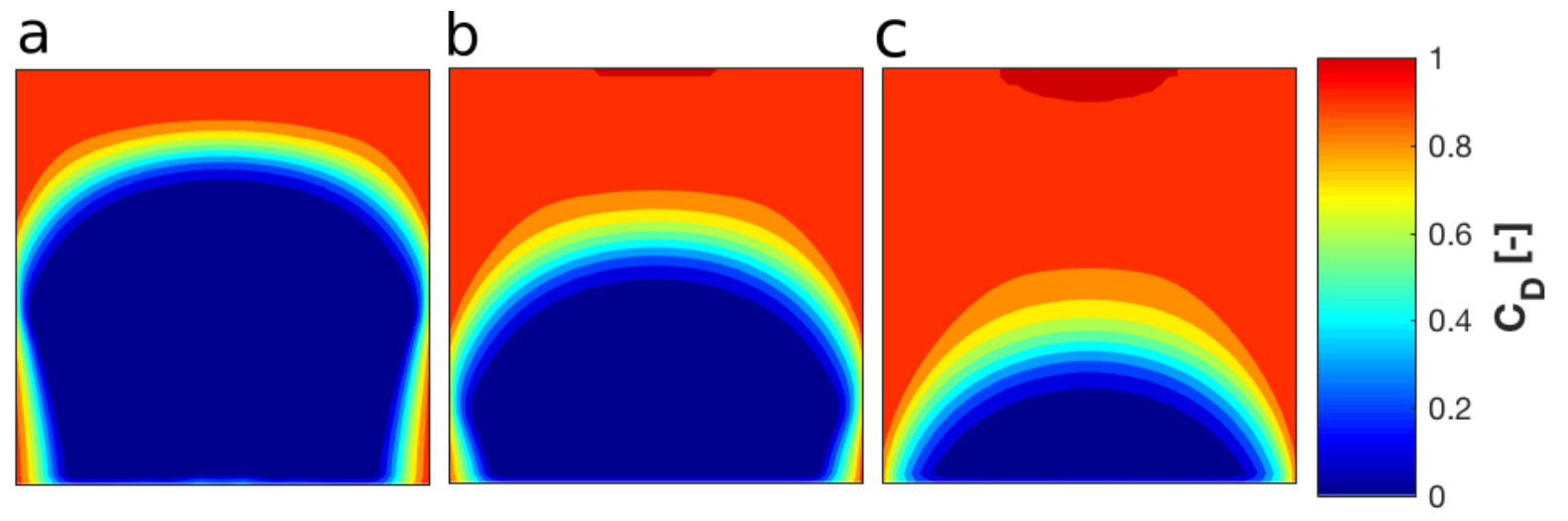

Figure 7 - Solvent concentration profile through the centre of the model (vertical plane) showing the typical drainage pattern using a hot solvent with $P e=10, \mu_{D}=1000, \rho_{D}=0.6$, 460 $n=2, \mu_{T}=1000, L e=10, m=4$ at a) $\left.\left.t_{D}=0.001, b\right) t_{D}=0.0015 \& c\right) t_{D}=0.002$. 

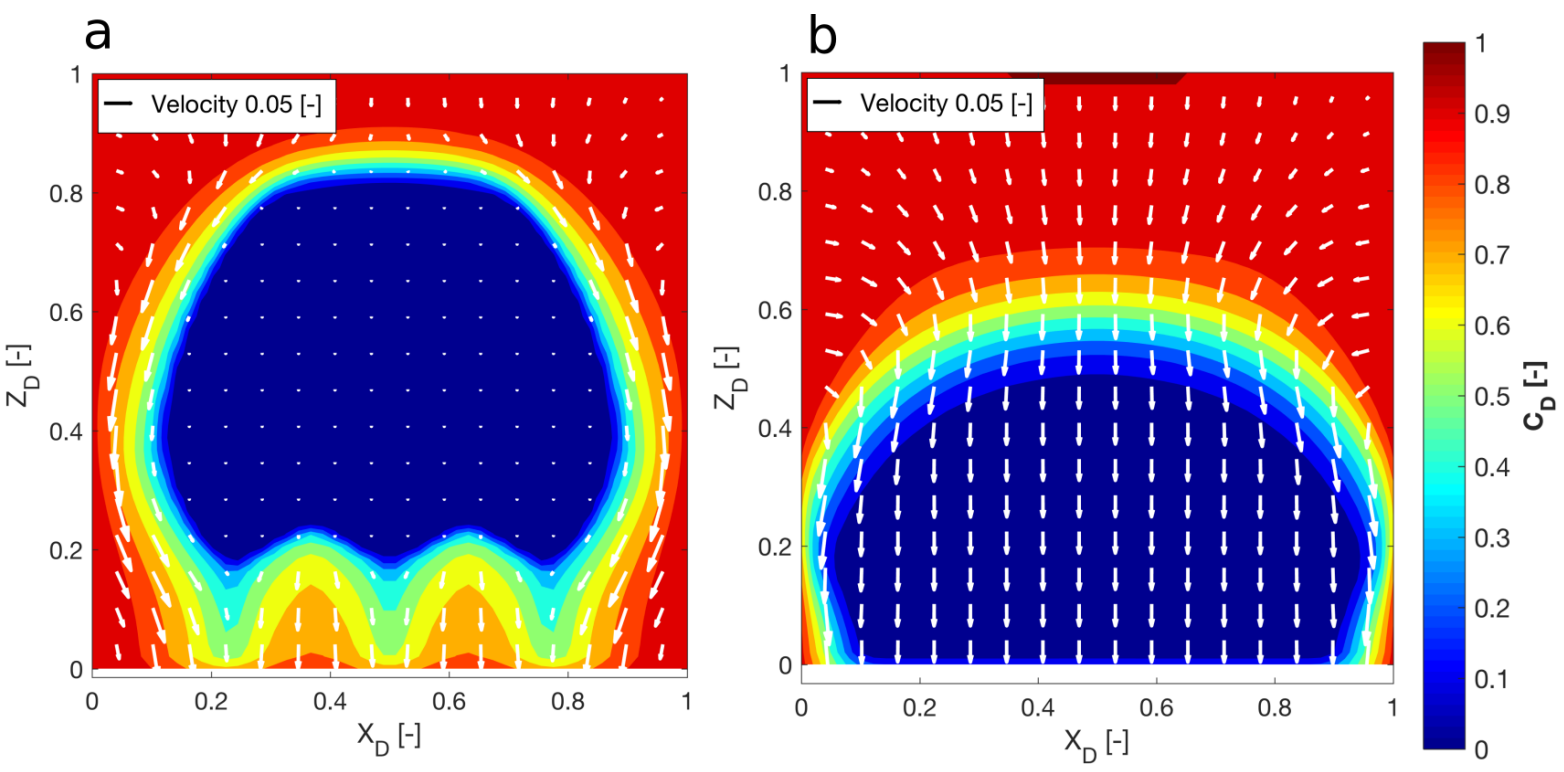

461

Figure 8 -Quiver plots showing the flow velocity through the centre of the matrix block (vertical plane) during recovery using a) cold solvent with $P e=10, \mu_{D}=1000, \rho_{D}=0.6$, 464 $n=2$ at $\left.t_{D}=0.01 \& b\right)$ hot solvent with $\mu_{T}=1000$, Le $=10, m=4$ at $t_{D}=0.0015$.

Figure 9 compares the oil recovery time and drainage profile of the matrix block for cold and transport inside the matrix block.
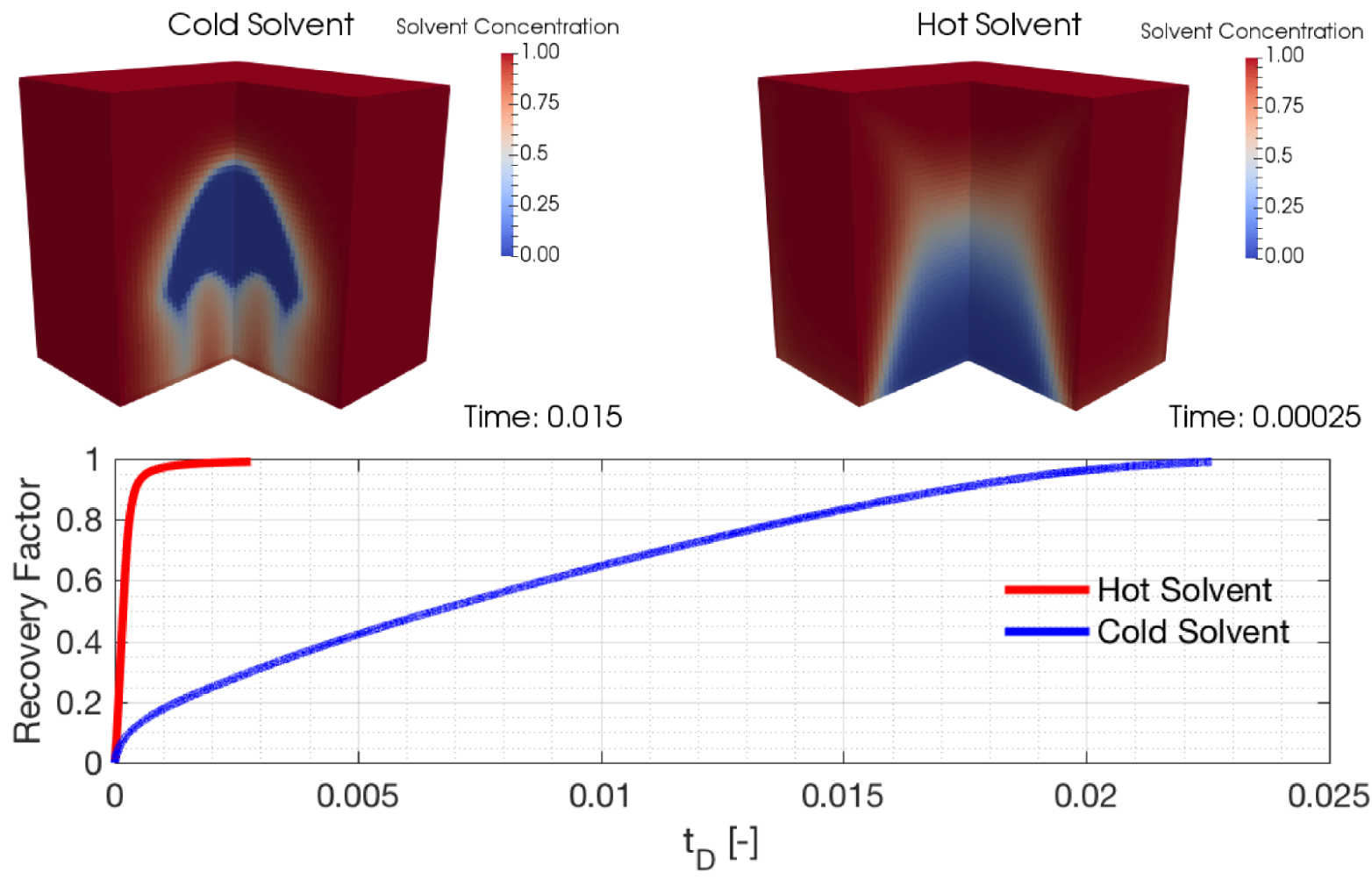
Figure 9 - Comparison of $3 D$ drainage profiles and recovery curves using a cold solvent with $P e=10, \mu_{D}=1000, \rho_{D}=0.6 \& n=2$ and a hot solvent with Le $=100, \mu_{T}=1000$ $\& m=4$. A $3 D$ video in avi format is provided comparing drainage profiles and recovery curves using a cold solvent and a hot solvent.

In order to analyse the effect of heat diffusion on the mass transfer and recovery process, a sensitivity analysis of Lewis numbers was conducted. Figure 10 shows that increasing the Lewis number, $L e$, increases the rate of oil recovery. This is a result of increased heat conduction from the solvent in the fractures into the matrix block which is quicker than solvent diffusion. Therefore, heat diffusion reduces the viscosity of the oil in the centre of the block earlier than solvent penetration to that depth.

The effect of Lewis number is greater at higher temperatures when the oil viscosity decreases more greatly, i.e at high values of $\mu_{T}$. An increase in the Lewis number results in the centre of the matrix block being heated quicker and therefore mobilises the resident oil more quickly explaining the increase in rate of oil recovery. Figure 10 also shows that as the Lewis number increases there is a big change in oil recovery time at lower values of Lewis number (in this example from 10 to 100), and then the change in oil recovery time becomes smaller (from 100 to 1000). If the Lewis number is large, the temperature in the centre of the block is raised considerably before any significant mass transfer has occurred. Raising the Lewis number further has little effect because the magnitude of the difference between mass and heat transfer.

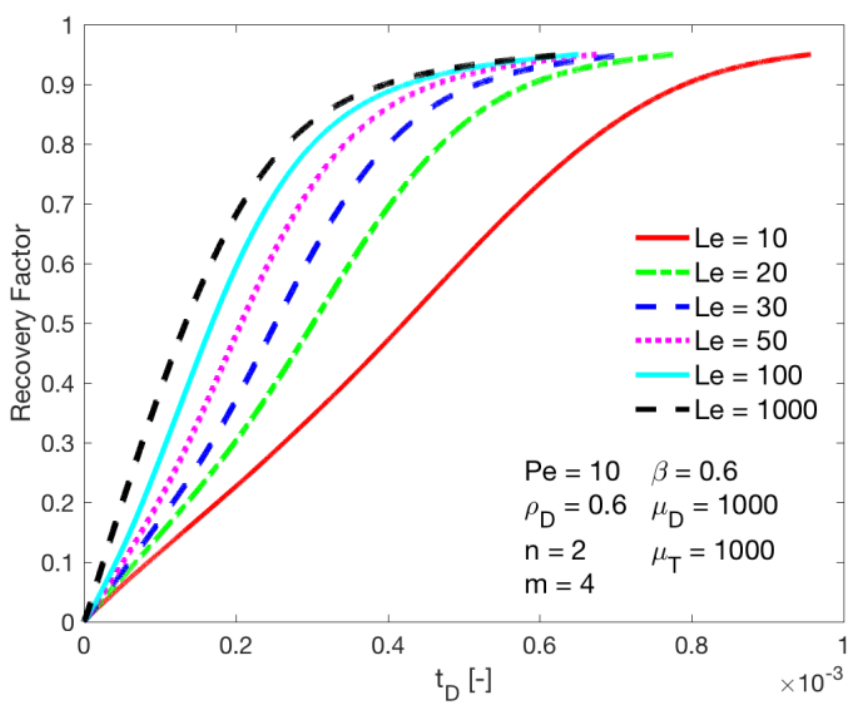


Figure 10 - Recovery curves for different values of the Lewis number.

491 Temperature profiles within the matrix block are shown in Figure 11 for one of the most highly 492 convective models in this study with $P e=10, \mu_{D}=1000, \rho_{D}=0.6, n=2, \mu_{\mathrm{T}}=1000$, 493 Le $=100, m=4$. The symmetrical temperature profiles over time show that heat transfer is dominated by conduction and there is very little convective heat transfer. It is important to also realise that the profile $\mathrm{c}$ is at $t_{D}=3 \times 10^{-5}$ when the recovery factor is still below 0.1 as 496 shown in Figure 10.
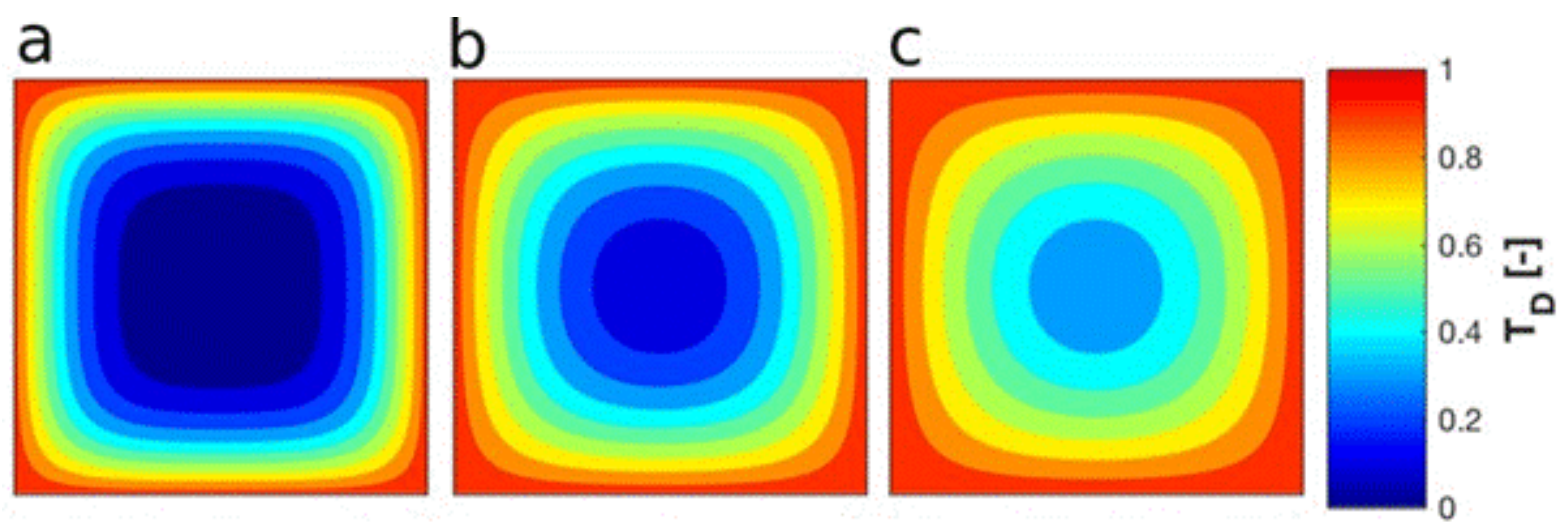

497

Figure 11 - Temperature profiles through the centre of the matrix block (vertical plane) at a) $t_{D}=1 \times 10^{-5}$, b) $t_{D}=2 \times 10^{-5} \&$ c) $t_{D}=3 \times 10^{-5}$ using Le=100 and $P e=10, \mu_{D}=$ 1000, $\rho_{D}=0.6, n=2, \mu_{T}=1000 \& m=4$

500 The Lewis number is unlikely to be less than 10 in typical oil reservoirs and Figure 12 shows

501 that this lower limit still displays a dominance of conductive heat transfer. Changes in 502 properties such as specific heat capacities, thermal conductivities and densities are therefore unlikely to shift the system from conduction to convective dominated heat transfer. For this reason, the thermal properties of the rock and liquids are not varied in this study. 


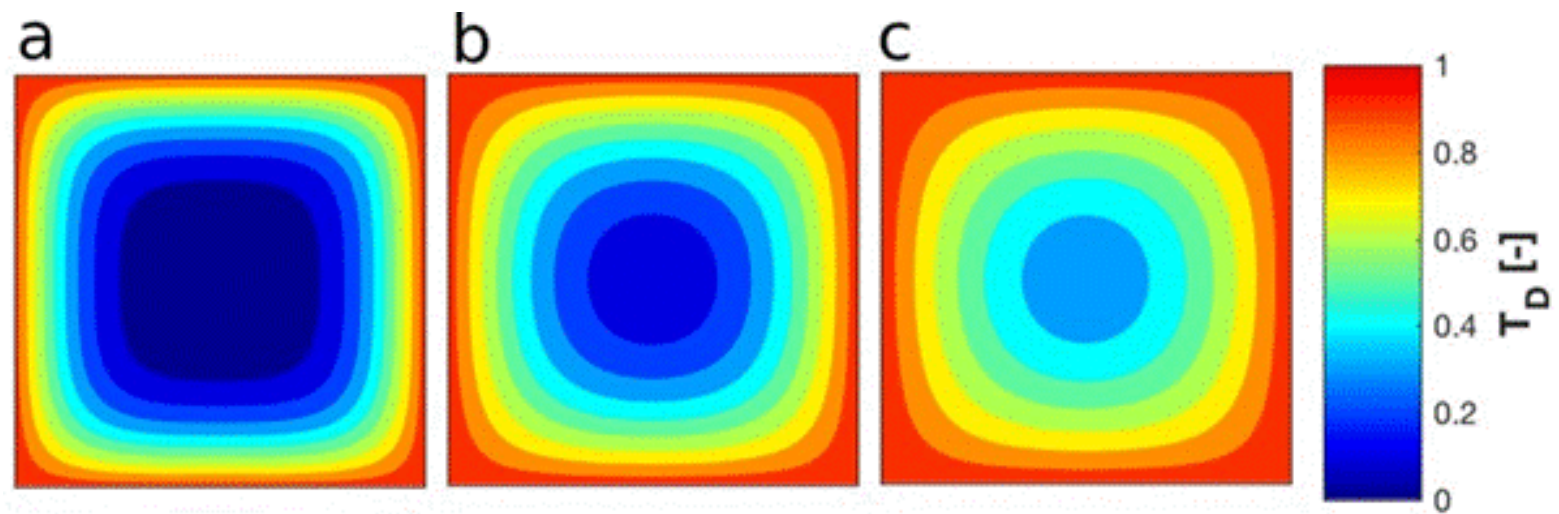

505

506

507

508

509

510

511

512

513

514

515

516

517

Figure 12 - Temperature profiles through the centre of the matrix block (vertical plane) at a) $t_{D}=1 \times 10^{-4}$, b) $t_{D}=2 \times 10^{-4}$, \& c) $t_{D}=3 \times 10^{-4}$, using Le=10 and $P e=10, \mu_{D}=$ $1000, \rho_{D}=0.6, n=2, \mu_{T}=1000 \& m=4$.

The viscosity relationship uses an exponent $m$ which usually has the value of 3-4 for heavy oilsolvent systems and it is determined experimentally. The recovery curves in Figure 13 show that the values of this exponent has very little effect on oil recovery processes through hot solvent injection.

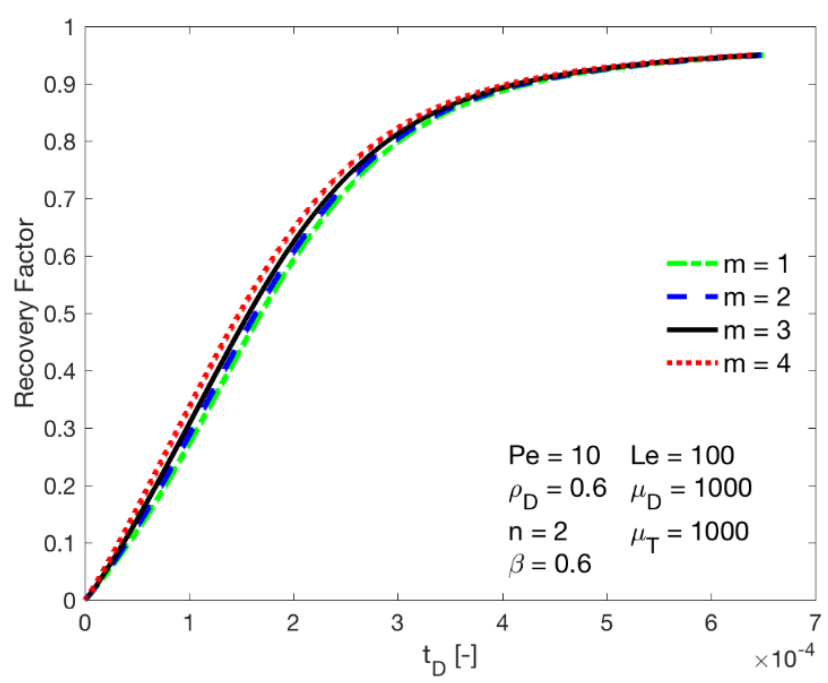

Figure 13 - Recovery curves for different values of exponent $m$ in the viscosity relationship.

\subsection{Solvent injection temperature optimisation algorithm}

The main purpose of this work is to develop a method by which the applicability of a hot solvent assisted gravity drainage process in a naturally fractured reservoir can be assessed without the need of complex simulations and experiments. This should act as a method of 
screening solvents and/or injection temperature ranges for potential oil recovery projects to highlight if the process warrants further investigation or not.

The model was ran for different parameters $\left(P e, \mu_{D}, \mu_{T}\right)$ while recording the dimensionless time required to reach a recovery factor of 0.8 . This data is plotted as a series of filled contour 522 plots in Figure 14 where each plot is for a specific value of viscosity ratio, $\mu_{D}$. The colour of any point on the figure represents the dimensionless time required to reach a recovery factor of 0.8 for a specific value of the Péclet number and the dimensionless thermal viscosity reduction term, $\mu_{T}$. These graphs clearly show that high Péclet numbers and high dimensionless thermal viscosity reduction terms reduce the oil recovery time. The blue region in the bottom left hand corners show the pure diffusion region where convective mass transfer is negligible and therefore the dimensionless thermal viscosity reduction term has very little or no effect on recovery time. As the Péclet number becomes slightly larger, the mass transport may still be diffusion dominated using a cold solvent. However, as the dimensionless viscosity reduction term increases it enhances convective mass transfer. At high Péclet numbers, convective mass transfer is dominant even when cold solvent is used and as the dimensionless viscosity reduction term increases, this dominance further increases.

The developed optimisation algorithm is shown in Figure 15 and aims to quickly determine qualitatively if increased injection temperature has any effect on oil recovery in addition to quantitatively estimating recovery times or target injection temperatures. The first step is to select a solvent considering that low solvent density and viscosity increase the recovery rate.

538 Next the dimensionless parameters Péclet number, $P e$, and viscosity ratio, $\mu_{D}$, can be calculated using the matrix and fluid properties. Once these are known, the corresponding graph from Figure 14 can be found. At this point the graphs can be used in two separate ways.

541 Firstly, if the proposed solvent injection temperature is known the viscosity reduction term can

542 be calculated from the oil properties using empirical relationships or experimental data. Using 
543 these values the time required to reach a recovery factor of 0.8 can be read from the

544 optimisation graph. Otherwise, if there is a target time to reach a recovery factor of 0.8 the

545 thermal viscosity reduction term required to achieve this target recovery time can be read from

546 the optimisation graph. This can be used to determine the injection temperature required to

547 achieve this recovery factor from the oil properties using empirical relationships or

548 experimental data. A similar approach could also be taken to prepare graphs similar to Figure

54914 but for a different recovery factor.

550 If the calculated Péclet number is beyond the range of the graph for the calculated viscosity

551 ratio, $\mu_{D}$ this also provides valuable information. If the Péclet is greater than the maximum on

552 the figure the cold solvent system demonstrates a convective dominated process with fast oil

553 recovery and increasing solvent injection temperature will further increase recovery rates.

554 However, for a Péclet number smaller than the minimum on the graph the solvent assisted oil

555 recovery process is diffusion dominated regardless of the dimensionless thermal viscosity

556 reduction term. 

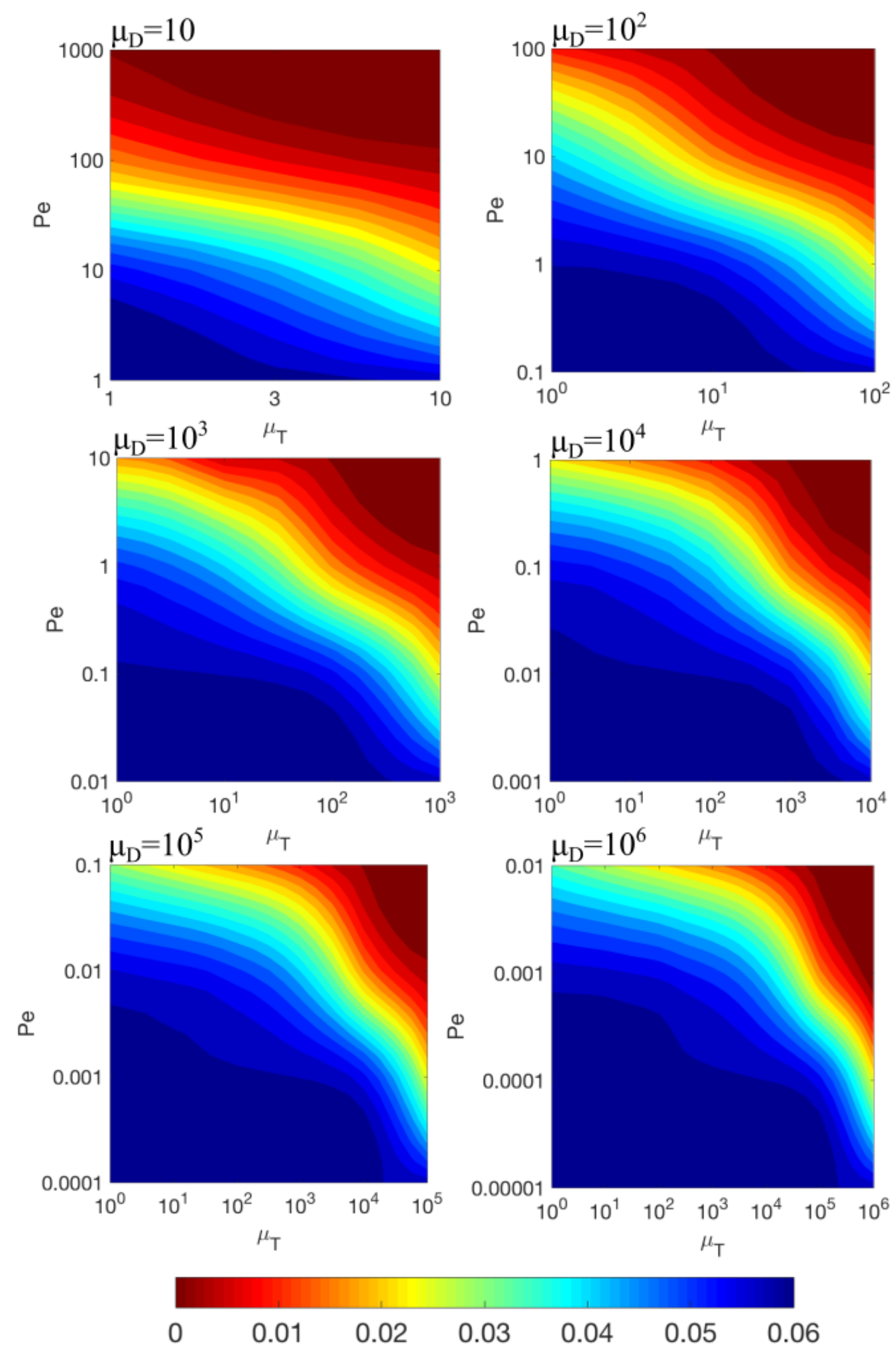
$t_{D}$ to reach $R F=0.8$

559 Figure 14-Optimisation graphs showing the dimensionless time to reach a recovery factor of 5600.8 using average properties of $\rho_{D}=0.7, n=2, m=4$, Le$=375$ and different values of $\mu_{D}$. 
561 Previous studies have shown that other factors such as density ratio and mass diffusion 562 exponent also impact the oil recovery rate ${ }^{46}$. This study has also shown that recovery rate is 563 impacted by the Lewis number and thermal viscosity exponent. However, these factors have a 564 smaller impact on the recovery rate and although it is important to consider them in our 565 simulations, to assess the feasibility of the process it is accurate enough to use average values.

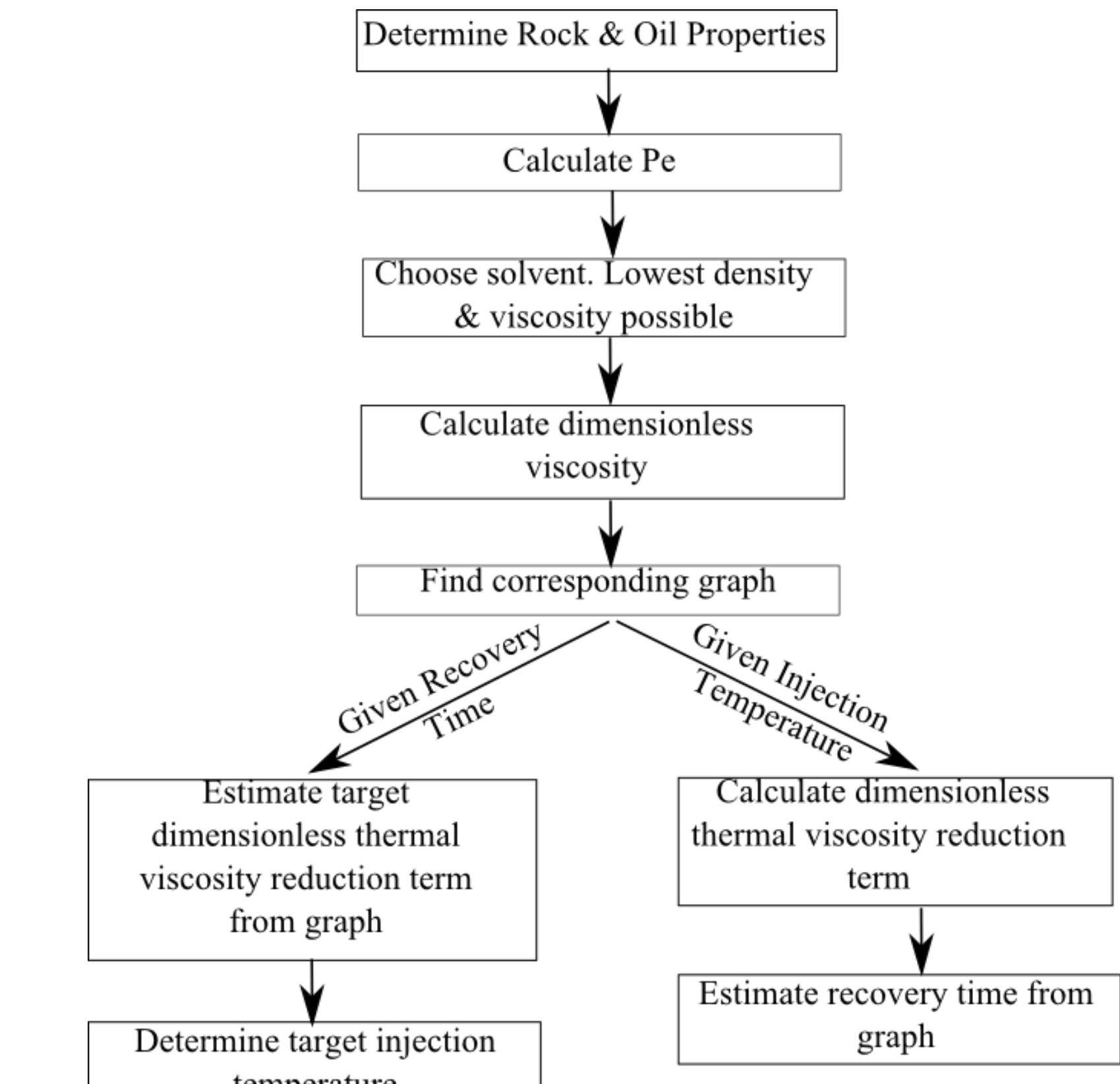
temperature

567 Figure 15 - Algorithm for determining optimum solvent and temperature of injection 
568 As previously mentioned, hot solvent injection is unlikely to be used as the sole method of recovery. Hot solvent may be injected initially to mobilise oil and then another phase such as $\mathrm{CO}_{2}$ or water may be injected afterwards reducing the volume of expensive solvent required.

571 The optimisation results in this study could be used to identify if the proposed solvent scheme

572 will initiate convective flow during the injection of the solvent or if the process will be diffusion dominated. Optimisation graphs for different recovery factors (e.g. 30\%, 50\%, 70\% etc.) can be generated depending on the desired recovery time or the equivalent volume of solvent available. Then after models can be simulated with optimised values (solvent temperatures and different recovery factors) followed by the injection of the second phase (hybrid processes) to compare the total recovery factor and thermal efficiency of the process. In these cases it is likely that there will be a target temperature to reach at the middle of the matrix block before changing the injected phase. This can be considered for further studies with focus on the temperature profile inside the matrix block and optimisation of multiphase flow.

\section{Conclusion}

In this study we focused on the use of hot solvents for oil recovery from naturally fractured heavy oil reservoirs which has been proposed to eliminate the issues associated with the complex structure of this type of reservoir. Simultaneous heat and mass transfer during oil recovery processes from fractured reservoirs was modelled. A concentration dependant mass diffusion coefficient was used to model mass transfer between liquid solvent and oil. This model illustrated that in the hot solvent injection process, heat diffusion is fast compared to mass transfer, therefore oil viscosity can be reduced drastically and can result in an increase in the dominance of convective mass transfer thereby increasing recovery rates. Through investigation of flow mechanisms and petrophysical reservoir properties, we demonstrated that when matrix permeability is very low increasing the injected solvent temperature has little or 
significant impact on the oil recovery. The recovery rate is largely controlled by the Péclet number, the ratio between solvent and oil viscosities and the ratio between hot and cold oil viscosities.

The other main outcome of this work was the development of a solution for the fast assessment of the applicability of a hot solvent assisted gravity drainage process in a naturally fractured reservoir. Through such solution, screening solvents and/or injection temperature ranges for potential oil recovery projects can be performed. This helps with designing appropriate hot solvent assisted gravity drainage processes for recovery from heavy oil fractured reservoirs. This was summarised in optimisation graphs showing the relative impact on recovery rates for various reservoir, oil and injected solvent properties.

\section{Appendix}

\section{A.1 Discretised equations}

The governing equations detailed in section 2 must be discretised before solving numerically. The momentum equation, Equation (30), is expressed in a discretised form as Equation (A.1). The spatial derivatives have been expressed using first order finite difference approximations. As there is no temporal derivative in this equation, this can be solved at any time as long as the viscosities and densities are known which are dependent on solvent concentration and temperature.

$\frac{\left.\frac{1}{\mu_{m i x, D}}\right|_{i+\frac{1}{2}, j, k}\left(\frac{P_{D_{i+1, j, k}}^{t}-P_{D_{i, j, k}}^{t}}{\Delta x_{D}}\right)+\left.\frac{1}{\mu_{m i x, D}}\right|_{i-\frac{1}{2}, j, k}\left(\frac{P_{D_{i-1, j, k}}^{t}-P_{D_{i, j, k}}^{t}}{\Delta x_{D}}\right)}{\Delta x_{D}}+$ $\frac{\left.\frac{1}{\mu_{m i x, D}}\right|_{i, j+\frac{1}{2}, k}\left(\frac{P_{D_{i, j+1, k}}^{t}-P_{D_{i, j, k}}^{t}}{\Delta y_{D}}\right)+\left.\frac{1}{\mu_{m i x, D}}\right|_{i, j-\frac{1}{2}, k}\left(\frac{P_{D_{i, j-1, k}}^{t}-P_{D_{i, j, k}}^{t}}{\Delta y_{D}}\right)}{\Delta y_{D}}+$

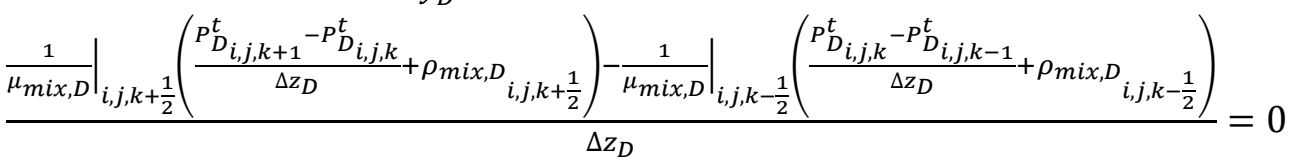

The solvent concentration equation, Equation (20), is expressed in a discretised form as Equation (A.2). The spatial derivatives have been expressed using first order finite difference 
617 at the previous time step. In the diffusive terms the solvent concentration appears before the

618 spatial derivative terms. To remove the non-linearity this term is evaluated at the previous time 619 step $(t)$ and the spatial derivatives are evaluated at the next time step $(t+\Delta t)$.

620

$\frac{C_{D_{i, j, k}}^{t+\Delta t}-C_{D_{i, j, k}}^{t}}{\Delta t_{D}}=\frac{\left.\left(C_{D}^{t}\right)^{n}\right|_{i+\frac{1}{2}, j, k}\left(\frac{C_{D_{i+1, j, k}}^{t+\Delta t}-C_{D_{i, j, k}}^{t+\Delta t}}{\Delta x_{D}}\right)+\left.\left(C_{D}^{t}\right)^{n}\right|_{i-\frac{1}{2}, j, k}\left(\frac{c_{D_{i-1, j, k}}^{t+\Delta t}-C_{D_{i, j, k}}^{t+\Delta t}}{\Delta x_{D}}\right)}{\Delta x_{D}}+$

621

$\frac{\left.\left(C_{D}^{t}\right)^{n}\right|_{i, j+\frac{1}{2}, k}\left(\frac{C_{D_{i, j+1, k}^{t}+\Delta t}^{t+\Delta C_{D}}-C_{i, j, k}^{t+\Delta t}}{\Delta y_{D}}\right)+\left.\left(C_{D}^{t}\right)^{n}\right|_{i, j-\frac{1}{2}, k}\left(\frac{C_{D_{i, j-1, k}}^{t+\Delta t}-C_{D_{i, j, k}}^{t+\Delta t}}{\Delta y_{D}}\right)}{\Delta y_{D}}+$

622

$\frac{\left.\left(C_{D}^{t}\right)^{n}\right|_{i, j, k+\frac{1}{2}}\left(\frac{c_{D_{i, j, k+1}+\Delta t}-C_{D_{i, j, k}}^{t+\Delta t}}{\Delta z_{D}}\right)+\left.\left(C_{D}^{t}\right)^{n}\right|_{i, j, k-\frac{1}{2}}\left(\frac{c_{D_{i, j, k-1}}^{t+\Delta t}-C_{D_{i, j, k}}^{t+\Delta t}}{\Delta z_{D}}\right)}{\Delta z_{D}}+$

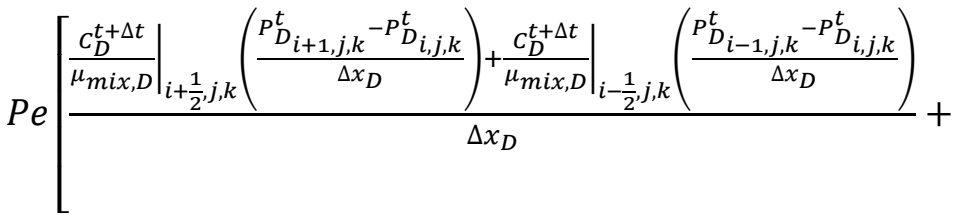

624

$\frac{\left.\frac{C_{D}^{t+\Delta t}}{\mu_{m i x, D}}\right|_{i, j+\frac{1}{2}, k}\left(\frac{P_{D_{i, j+1, k}^{t}}^{t}-P_{D_{i, j, k}}^{t}}{\Delta y_{D}}\right)+\left.\frac{C_{D}^{t+\Delta t}}{\mu_{m i x, D}}\right|_{i, j-\frac{1}{2}, k}\left(\frac{P_{D_{i, j-1, k}}^{t}-P_{D_{i, j, k}}^{t}}{\Delta y_{D}}\right)}{\Delta y_{D}}+$

625

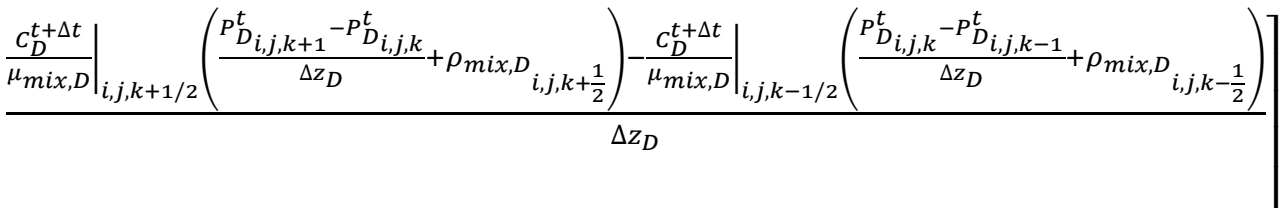

626 The heat equation, Equation (41), is expressed in a discretised form as equation (A.3). The

627 spatial derivatives have been expressed using first order finite difference approximations and

628 a forwards time difference is used. The pressure derivatives are also evaluated at the previous

629 time step.

630

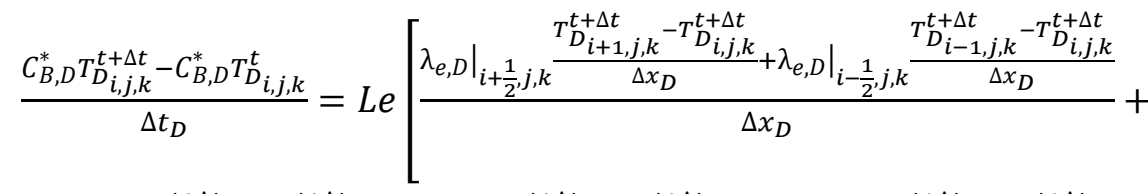

631

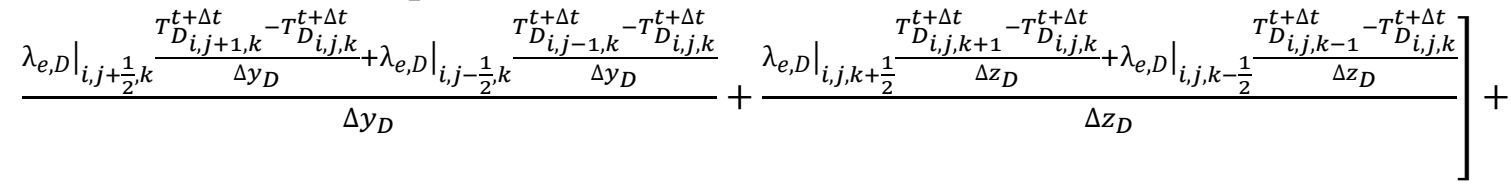




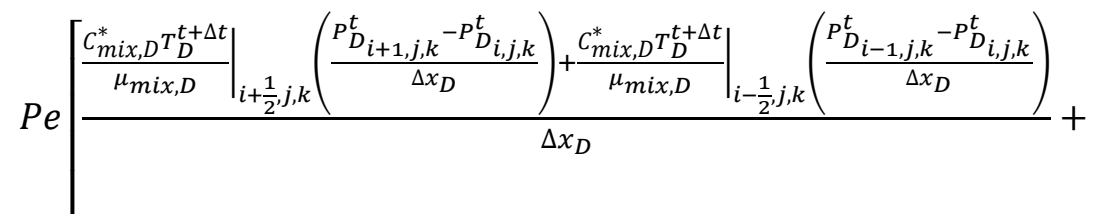

633

$\frac{\left.\frac{C_{m i x, D}^{*} T_{D}^{t+\Delta t}}{\mu_{m i x, D}}\right|_{i, j+\frac{1}{2}, k}\left(\frac{P_{D_{i, j+1, k}}^{t}-P_{D_{i, j, k}}^{t}}{\Delta y_{D}}\right)+\left.\frac{C_{m i x, D}^{*} T_{D}^{t+\Delta t}}{\mu_{m i x, D}}\right|_{i, j-\frac{1}{2}, k}\left(\frac{P_{D_{i, j-1, k}}^{t}-P_{D_{i, j, k}}^{t}}{\Delta y_{D}}\right)}{\Delta y_{D}}+$

$$
\left.\left.\frac{\left.\frac{C_{m i x, D}^{*} T_{D}^{t+\Delta t}}{\mu_{m i x, D}}\right|_{i, j, k+1 / 2}\left(\frac{P_{D_{i, j, k+1}}^{t}-P_{D_{i, j, k}}^{t}+\rho_{m i x, D}}{\Delta z_{D}}\right)-\left.\frac{C_{m i x, D}^{*} T_{D}^{t+\Delta t}}{\mu_{m i x, D}}\right|_{i, j, k-1 / 2}\left(\frac{P_{D_{i, j, k}}^{t}-P_{D_{i, j, k-1}}^{t}}{\Delta z_{D}}+\rho_{m i x, D}\right.}{\Delta z_{D}}\right)\right]
$$

635

636

637

638

639

640

641

$\theta_{i+\frac{1}{2}, j, k}=\left\{\begin{array}{rc}\theta_{i+1, j, k} & \text { if } \quad P_{i+1, j, k} \geq P_{i, j, k} \\ \theta_{i, j, k} & \text { otherwise }\end{array}\right.$

\section{A.2 Evaluating properties at cell boundaries} (A.4),

The discretised equations (A.1-A.3) all include terms that are evaluated at the boundaries of cells. In this study a single point upwind selection is used to determine what value to assign at the boundaries. This requires the direction of flow on each boundary. Considering the value $\theta$ at the cell face in a vertical plane, such as $i+\frac{1}{2}, j, k$, this is evaluated by the criteria in Equation

In the vertical direction this is slightly more complex and the value $\theta$ at the cell face $i, j, k+\frac{1}{2}$ is evaluated using the criteria in Equation (A.5).

$644 \quad \theta_{i, j, k+\frac{1}{2}}=\left\{\begin{array}{ccc}\theta_{i+1, j, k} & \text { if } & \frac{P_{D_{i, j, k+1}}-P_{D_{i, j, k}}}{\Delta z_{D}}>-\rho_{\text {mix }, D_{i, j, k+1}} \\ \theta_{i, j, k} \quad \text { if } & \frac{P_{D_{i, j, k+1}}-P_{D_{i, j, k}}}{\Delta z_{D}}<-\rho_{\text {mix }, D_{i, j, k}} \\ \text { else there is no vertical flow across cell face }\end{array}\right.$

If there is no vertical flow across the cell face the terms for momentum, mass or heat flux across that boundary can be ignored for the cell being considered.

647 The values at the boundaries require the flow direction which is unknown at the next time level.

648 Therefore the flow direction from the previous time step is always used. This means that when 649 solving for the concentration at $t+\Delta t$, the flow directions at time $t$ are used instead. 
650 A.3 Initial and boundary conditions

651 Isothermal initial conditions:

$652 \quad C_{D}=0$

$653 \quad T_{D}=1$

654 Isothermal boundary conditions:

$655 \quad P_{D_{f}}=\rho_{D}\left(1-z_{D}\right)$

$656 \quad C_{D_{f}}=1$

$657 \quad T_{D_{f}}=1$

$658 \quad \mu_{T}=1$

659 Thermal initial conditions:

$660 C_{D}=0$

$661 T_{D}=0$

662 Thermal boundary conditions:

$663 P_{D_{f}}=\rho_{D}\left(1-z_{D}\right)$

$664 C_{D_{f}}=1$

$665 T_{D_{f}}=1$ 


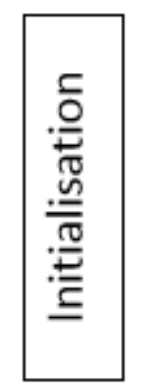

i. Set all initial variables

ii. Create computational grid

iii. Assign $C_{D_{i, j, k}}^{t=0}$ and $T_{D_{i, j, k}}^{t=0}$

iv. Update fluid properties in all cells

v. Solve initial pressure distribution $P_{D_{i, j, k}}^{t=0}$

1. Use $P_{D_{i, j, k}}^{t=n}$ and corresponding velocity field $\boldsymbol{U}^{t=n}$

2. Calculate $C_{D_{i, j, k}}^{t=n+1}$ using $\boldsymbol{U}^{t=n}$

3. Calculate $T_{D_{i, j, k}}^{t=n+1}$ using $\boldsymbol{U}^{t=n}$

4. Update all cell properties

5. Calculate $P_{D_{i, j, k}}^{t=n+1}$ using $C_{D_{i, j, k}}^{t=n+1}$ and $T_{D_{i, j, k}}^{t=n+1}$

6. $t_{D}=t_{D}+\Delta t_{D}$

7. Calculate recovery factor

8. Save data for time step

Notes

The authors declare no competing financial interest.

\section{Acknowledgements}

671 The authors would like to acknowledge the School of Engineering at the University of

672 Aberdeen for providing the required facilities to complete this research. We are also grateful

673 for technical comments from Mehrdad Taghizadeh Manzari (School of Geosciences at the 
674 University of Aberdeen). In addition to this, we appreciate the access to the Maxwell High

675 Performance Computing Cluster of the University of Aberdeen IT Service

676 (www.abdn.ac.uk/staffnet/research/hpc.php), provided by Dell Inc. and supported by Alces

677 Software. This research did not receive any specific grant from funding agencies in the public,

678 commercial, or not-for-profit sectors.

\section{References}

(1) Rassenfoss, S. Finding pathways to produce heavy oil from Canadian carbonates. JPT, J. Pet. Technol. 2013, 65, 58-67.

(2) Ezeuko, C. C.; Gates, I. D. Simulation Analysis of Steam-Based EOR Using MultiObjects Grosmont Models. Presented at the SPE Heavy Oil Conference-Canada, 11-13 June 2013, Calgary, Canada. 2013. Conference paper SPE-165484-MS.

(3) Butler, R. M.; McNab, G. S.; Lo, H. Y. Theoretical studies on the gravity drainage of heavy oil during in-situ heating. Can. J. Chem. Eng. 1981, 59, 455-460.

(4) Khan, M. A. B.; Mehrotra, A. X.; Svrcek, W. Y. Viscosity models for gas-free Athabasca bitumen. J. Can. Pet. Technol. 1984, 23, 47-53.

(5) Araújo, E. A.; Diniz, A. A. R.; Gurgel, A. R.; Lima, D. M. B. S.; Dutra, T. V.; Barillas, J. L. M. Analysis of oil production by applying in situ combustion. Pet. Sci. Technol. 2016 34, 50-55.

(6) Bybee, K. Comparison of Cyclic Steam Stimulation and Steam-Assisted Gravity Drainage. JPT, J. Pet. Technol. 2003, 55, 68-69.

(7) Butler, R. M.; Stephens, D. J. The gravity drainage of steam-heated oil to parallel horizontal wells. J. Can. Pet. Technol. 1981, 20, 90-96.

(8) Tavallali, M.; Maini, B.; Harding, T.; Busahmin, B. Assessment of SAGD Well Configuration Optimization in Lloydminster Heavy Oil Reserve. Presented at the SPE/EAGE European Unconventional Resources Conference and Exhibition, 20-22 March 2012, Vienna, Austria. 2012. Conference paper SPE 153128.

(9) Nasr, T.; Isaacs, E. Process for enhancing hydrocarbon mobility using steam additive. US Patent 6230814, 2001.

(10) Nasr, T. R.; Ayodele, O. R. New hybrid steam-solvent processes for the recovery of heavy oil and bitumen. Presented at the Abu Dhabi International Petroleum Exhibition and Conference, 5-8 November 2006, Abu Dhabi, UAE. 2006. Conference paper SPE-101717MS.

(11) Gates, I. Solvent-aided Steam-Assisted Gravity Drainage in thin oil sand reservoirs. J. Pet. Sci. Eng. 2010, 74, 138-146. 
(12) Al-Gosayir, M.; Babadagli, T.; Leung, J.; Al-Bahlani, A. M. In-situ recovery of heavy-oil from fractured carbonate reservoirs: Optimization of steam-over-solvent injection method. $J$. Pet. Sci. Eng. 2015, 130, 77-85.

(13) Zhao, L. Steam alternating solvent process. SPE Reservoir Eval. Eng. 2007, 10, 185-190.

(14) Gupta, S.; Gittins, S.; Picherack, P. Field implementation of solvent aided process. J. Can. Pet. Technol. 2005, 44, 8-13.

(15) Zirrahi, M.; Hassanzadeh, H.; Abedi, J. Experimental and modeling studies of MaxKay River bitumen and water. J. Pet. Sci. Eng. 2017, 151, 305-310.

(16) Zirrahi, M.; Hassanzadeh, H.; Abedi, J. Experimental and modelling studies of water, light nalkenes and MacKay River bitumen ternary systems. Fuel, 2017, 196, 1-12.

(17) Azinfar, B.; Haddadni, A.; Zirrahi, M.; Hassanzadeh, H.; Abedi, J. Effect of Asphaltene on Phase Behaviour and Thermophysical Properties of Solvent/Bitumen Systems. J. Chem. Eng. Data. 2017, 62, 547-557.

(18) Dickson, J. L.; Subramanian, G.; Shah, P.; Otahal, J. M.; Dittaro, L. M.; Jaafar, A. E.; Yerian, J. A. Key Learnings from a Simulation Study of a Solvent-Assisted SAGD Pilot at Cold Lake. presented at SPE Heavy Oil Conference-Canada, 11-13 June 2013, Calgary, Canada. 2013. Conference paper SPE-165486-MS.

(19) Dittaro, L. M.; Jaafar, A. E.; Perlau, D. L.; Boone, T. J.; Yerian, J. A.; Dickson, J. L.; Wattenbarger, R. C. Findings from a Solvent-Assisted SAGD Pilot at Cold Lake. Presented at the SPE Heavy Oil Conference-Canada, 11-13 June 2013, Calgary. 2013. Conference paper SPE-165434-MS.

(20) Azom, P. N.; Srinivasan, S. Modelling coupled heat transfer and multiphase flow during the expanding solvent steam-assisted gravity drainage (ES-SAGD) process. Presented at the SPE Annual Technical Conference and Exhibition, 30 September - 2 October 2013, New Orleans, USA. 2013. Conference paper SPE-166357-MS.

(21) Leyva-Gomez, H.; Babadagli, T.High-Temperature Solvent Injection for Heavy-Oil Recovery From Oil Sands: Determination of Optimal Application Conditions Through Genetic Algorithm. SPE Reservoir Eval. Eng. 2017, 20, 372-382.

(22) Sabet, N.; Hassanzadeh, H.; Abedi, J. Selection of efficient solvent in solvent-aided thermal recovery of bitumen. Chem. Eng. Sci. 2017, 161, 198-205.

(23) Khaledi, R.; Boone, T. J.; Motahhari, H. R.; Subramanian, G. Optimized Solvent for Solvent Assisted-Steam Assisted Gravity Drainage (SA-SAGD) Recovery Process. Presented at the SPE Canada Heavy Oil Technical Conference, 9-11 June 2015, Calgary, Canada. 2015. Conference paper SPE-174429-MS.

(24) Marciales, A.; Babadagli, T. Selection of Optimal Solvent Type for High-Temperature Solvent Applications in Heavy-Oil and Bitumen Recovery. Energy Fuels. 2016, 30, 25632573. 
(25) Hassanzadeh, H.; Faradonbeh, M. R.; Harding, T. Numerical Simulation of Solvent and Water Assisted Electrical Heating of Oil Sands Including Aquatermolysis and Thermal Cracking Reactions. AlChE J. 2017, 63, 4243-4258.

(26) Hascakir, B.; Babadagli, T.; Akin, S. Experimental and Numerical Modeling of Heavy-Oil Recovery by Electrical Heating. Presented at the 2008 SPE International Thermal Operations and Heavy Oil Symposium 20-23 October 2008, Calgary, Canada. 2008. Conference Paper SPE-117669-MS.

(27) Hascakir, B.; Babadagli, T.; Akin, S. Field-Scale Analysis of Heavy-Oil Recovery by Electrical Heating. SPE Reservoir Eval. Eng. 2010, 13, 131-142.

(28) Vinegar, H. J.; Picha, M. G.; Schoeling, L. G. Thermal process for subsurface formations US Patent 7942203, 2011.

(29) Pathak, V.; Babadagli, T.; Edmunds, N. Experimental investigation of bitumen recovery from fractured carbonates using hot solvents. J. Can. Pet. Technol. 2013, 52, 289-295.

(30) Leyva-Gomez, H.; Babadagli, T. Hot Solvent Injection for Heavy Oil/Bitumen Recovery from Fractured Reservoirs: An Experimental Approach To Determine Optimal Application Conditions. Energy Fuels. 2016, 30, 2780-2790.

(31) Kahrobaei, S.; Farajzadeh, R.; Suicmez, V. S.; Bruining, J. Gravity-enhanced transfer between fracture and matrix in solvent based enhanced oil recovery. Ind. Eng. Chem. Res. 2012, 51, 14555-14565.

(32) Telmadarreie, A.; Trivedi, J. Evaluation of foam generated with the hydrocarbon solvent for extra-heavy oil recovery from fractured porous media: Pore-scale visualization. J. Pet. Sci. Eng. 2017, 157, 1170-1178.

(33) Telmadarreie, A.; Trivedi, J. New Insight on Carbonate-Heavy-Oil Recovery: Pore-Scale Mechanisms of Post-Solvent Carbon Dioxide Foam/Polymer-Enhanced-Foam Flooding. SPE J. (Soc. Pet. Eng.) 2016, 21, 1655-1668.

(34) Emrani, A. S.; Nasr-El-Din, H. A. An experimental study of nanoparticale-polymer-stabilized CO2 foam. Colloids Surf. A. 2017, 524, 17-27.

(35) Rafati, R.;Sharifi Haddad, A.; Hamidi, H. Experimental study on stability and rheological properties of aqueous foam in the presence of reservoir natural solid particles. Colloids Surf., A. 2016, 509, 19-31.

(36) Edmunds, N.; Barrett, K.; Solanki, S.; Cimolai, M. Prospects for commercial bitumen recovery from the Grosmont carbonate, Alberta. J. Can. Pet. Technol. 2009, 48, 26-32.

(37) Mohebati, M. H.; Yang, D.; MacDonald, J. Thermal Recovery of Bitumen From the Grosmont Carbonate Formation - Part 1: The Saleski Pilot. J. Can. Pet. Technol. 2014, 53, 200-211. 
(38) Yang, D.; Mohebati, M. H.; Brand, S.; Bennett, C. Thermal recovery of bitumen from the grosmont carbonate formation - Part 2: Pilot interpretation and development stratergy. J. Can. Pet. Technol. 2014, 53, 212-223.

(39) Sharifi Haddad, A.; Gates, I. CO2-based heavy oil recovery process for post-CHOPS reservoirs. J. CO2 Utilization. 2017, 19, 238-246.

(40) Da Silva, F. V.; Belery, P. Molecular Diffusion in Naturally Fractured Reservoirs: A Decisive Recovery Mechanism. Presented at SPE Annual Technical Conference and Exhibition, 8-11 October 1989, San Antonio, Texas, USA. 1989. Conference paper SPE-19672-MS.

(41) Trivedi, J.; Babadagli, T. Efficiency of diffusion controlled miscible displacement in fractured porous media. Transp. Porous Med. 2008, 71, 379-394.

(42) Hatiboglu, C.; Babadagli, T. Diffusion mass transfer in miscible oil recovery: Visual experiments and simulation. Transp. Porous Med. 2008, 74, 169-184.

(43) Warren, J. E.; Root, P. J. The behaviour of naturally fractured reservoirs. Soc. Pet. Eng. J. 1963, 3, 245-255.

(44) Faradonbeh, M. R.; Harding, T. G.; Abedi, J. The applicability of expanding solven steamassisted gravity drainage of heavy oil and bitumen, Part 1: Enhanced flow rate at mobile zone. Presented at the SPE Annual Technical Conference and Exhibition, 8-10 October 2012, San Antonio, Texas, USA. 2012. Conference paper SPE-160316-MS.

(45) Okazawa, T. Impact of concentration-dependance of diffusion coeffiecient on VAPEX drainage rates. J. Can. Pet. Technol. 2009, 48, 47-54.

(46) Sharifi Haddad, A.; Hejazi, S. H.; Gates, I. D. Modelling solvent enhanced gravity drainage from a single matrix block in fractured oil reservoirs. J. Pet. Sci. Eng. 2017, 152, 555-563.

(47) Pooladi-Darvish, M.; Tortike, W. S.; Farouq Ali, S. M. Steam heating of fractured formation containing heavy oil: Basic premise and a single-block analytical model. Presented at the SPE Annual Technical Conference and Exhibition, 25-28 September 1994, New Orleans, USA. 1994. Conference paper SPE-28642-MS.

(48) Scott Fogler, H. Elements of Chemical Reaction Engineering. Prentice Hall PTR: London. 1990.

(49) Guan, J. G.; Kariznovi, M.; Nourozieh, H.; Abedi, J. Density and Viscosity for Mixtures of Athabasca Bitumen and Aromatic Solvents. J. Chem.Eng.Data. 2013, 58, 611-624.

(50) Luo, H.; Salama, D.; Kryuchkov, S.; Kantzas, A. The effect of Volume Changes Due to Mixing on Diffusion Coefficient Determination in Heavy Oil and Hydrocarbon Solvent System. Presented at the 2007 SPE Annual Technical Converence and Exhibition, 11-14 November 2007, Anaheim, California. 2007. Conference paper SPE 110522.

(51) Computer Modelling Group Ltd. STARS User Guide Version 2016. Calgary, Alberta, Canada. 
(52) Winterfeld, P. H.; Wu, Y. S. Simulation of Coupled Thermal/Hydrological/Mechanical Phenomena in Porous Media. SPE J.(Soc. Pet. Eng.) 2016, 21, 1041-1049.

(53) Guennebaud, G.; Jacob, B.; and others. Eigen v3. http://eigen.tuxfamily.org. 2010.

(54) Kim, M. C. Linear stability analysis on the onset of the Rayleigh-Taylor instability of a miscible slice in a porous medium. J. Eng. Math. 2015, 90, 105-118.

(55) Sharp, D. H. An overview of Rayleigh-Taylor instability. Physica D. 1984, 12, 3-10.

(56) Wilke, C. R.; Chang, P.Correlation of diffusion coefficients in dilute solutions. AlChE J. 1955, 1, 264-270.

\begin{tabular}{|c|c|c|}
\hline 680 & \multicolumn{2}{|c|}{ Nomenclature } \\
\hline 681 & $\mathrm{C}$ & Concentration [vol/vol] \\
\hline 682 & $\mathrm{C}^{*}$ & Volumetric heat capacity $\left[\mathrm{J} \cdot \mathrm{m}^{-3} \cdot \mathrm{K}^{-1}\right]$ \\
\hline 683 & $\mathrm{D}$ & Mass diffusion coefficient $\left[\mathrm{m}^{2} \mathrm{~s}^{-1}\right]$ \\
\hline 684 & $\mathrm{~g}$ & Acceleration due to gravity $\left[\mathrm{m}^{2} \cdot \mathrm{s}^{-1}\right]$ \\
\hline 685 & $\mathrm{~h}$ & Depth in fracture [m] \\
\hline 686 & $\mathrm{k}$ & Permeability $\left[\mathrm{m}^{2}\right]$ \\
\hline 687 & $\mathrm{~L}$ & Characteristic length [m] \\
\hline 688 & $\mathrm{P}$ & Pressure $[\mathrm{Pa}]$ \\
\hline 689 & $\mathrm{t}$ & Time $[\mathrm{s}]$ \\
\hline 690 & $\mathrm{~T}$ & Temperature $[\mathrm{K}]$ \\
\hline 691 & $\mathrm{U}$ & Flow velocity $\left[\mathrm{m} \cdot \mathrm{s}^{-1}\right]$ \\
\hline 692 & $\mathrm{~V}$ & Volume fraction [-] \\
\hline 693 & Xs & Lederer viscosity exponent [-] \\
\hline 694 & $\mathrm{X}$ & $\mathrm{x}$-coordinate $[\mathrm{m}]$ \\
\hline 695 & $\mathrm{y}$ & $\mathrm{y}$-coordinate $[\mathrm{m}]$ \\
\hline 696 & $\mathrm{Z}$ & $\mathrm{z}$-coordinate $[\mathrm{m}]$ \\
\hline 697 & Dir & sionless Parameters \\
\hline 698 & Le & Lewis number [-] \\
\hline 699 & $\mathrm{Pe}$ & Péclet number [-] \\
\hline 700 & $\mathrm{n}$ & Mass diffusion exponent [-] \\
\hline 701 & $\mathrm{~m}$ & Butler viscosity exponent [-] \\
\hline
\end{tabular}


$\mu_{D} \quad$ Dimensionless oil-solvent viscosity ratio [-]

$703 \quad \mu_{T} \quad$ Dimensionless thermal viscosity reduction [-]

$704 \rho_{D} \quad$ Dimensionless solvent-oil density ratio [-]

705 Greek

$706 \quad \alpha \quad$ Thermal diffusivity $\left[\mathrm{m}^{2} \mathrm{~s}^{-1}\right]$

$707 \quad \beta \quad$ Lederer viscosity parameter [-]

$708 \delta \quad$ Pore constrictivity [-]

$709 \phi \quad$ Porosity [-]

$710 \lambda \quad$ Thermal conductivity $\left[\mathrm{W} \cdot \mathrm{m}^{-1} \cdot \mathrm{K}^{-1}\right]$

$711 \mu \quad$ Dynamic viscosity [Pa.s]

$712 \rho \quad$ Density $\left[\mathrm{kg} \cdot \mathrm{m}^{-3}\right]$

$713 \tau \quad$ Pore tortuosity [-]

$714 v \quad$ Kinematic viscosity $\left[\mathrm{m}^{2} \cdot \mathrm{s}^{-1}\right]$

715 Subscripts

716 B Bulk

717 D Dimensionless

718 e Effective

719 f Fracture

720 hot Injected solvent conditions

$721 \mathrm{~m}$ Molecular

722 mix Mixture

$723 \quad$ o Oil

$724 \mathrm{R}$ Initial reservoir conditions

725 rock Matrix rock

726 S Solvent 

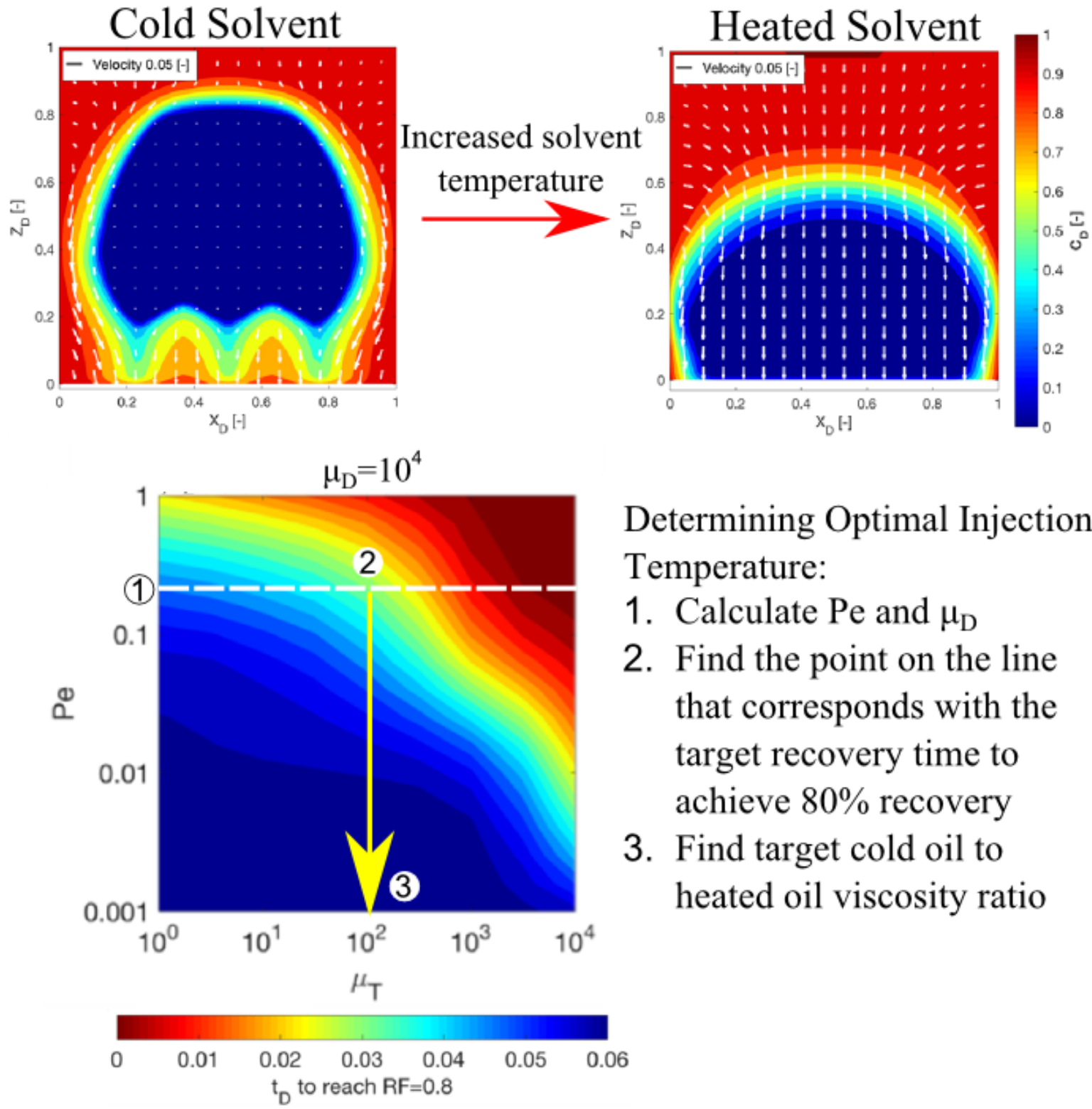

Determining Optimal Injection Temperature:

1. Calculate Pe and $\mu_{D}$

2. Find the point on the line that corresponds with the target recovery time to achieve $80 \%$ recovery

3. Find target cold oil to heated oil viscosity ratio 